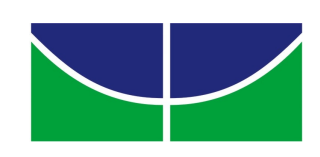

UNIVERSIDADE DE BRASÍLIA

INSTITUTO DE FÍSICA

PROGRAMA DE PÓS-GRADUAÇÃO EM FÍSICA

\title{
Estudo do comportamento termodinâmico de sistemas físicos com interação de longo alcance na proximidade da região crítica
}

\author{
Lilah Fialho de Lima Simões
}

Dissertação de Mestrado

Brasília

Fevereiro de 2017 


\section{Lilah Fialho de Lima Simões}

\section{Estudo do comportamento termodinâmico de sistemas físicos com interação de longo alcance na proximidade da região crítica}

Dissertação apresentada ao Instituto de Física da Universidade de Brasília, para a obtenção de Título de Mestre em Física Teórica.

Orientador: Prof. Dr. Marco A. Amato

\section{Brasília}


"Se você é neutro em situações de injustiça, você escolhe o lado do opressor"

- Desmond Tutu 
Dedicado a minha vó Maria Licinia 


\section{Agradecimentos}

Agradeço a minha família pelo companheirismo e pelo reconhecimento. Aos meus amigos de trabalho que me aguentam todo dia, principalmente por me lembrarem sempre o quão maravilhoso e terrível, ao mesmo tempo, é estudar Física.

Agradeço às minhas amigas Flávia e Gabriela e ao meu amigo Murilo por serem sempre um porto seguro. Ao meu namorado Daniel pelo apoio diário e por tentar me mostrar o mundo de forma mais positiva.

Agradeço ao meu orientador pela paciência, ajuda e conselhos, não somente quanto à Física, e por me incentivar a olhar de uma forma crítica os fenômenos ao meu redor. E ao $\mathrm{CNPq}$, pelo apoio financeiro. 


\section{Resumo}

O estudo de transição de fases de sistemas termodinâmicos na proximidade do ponto crítico é desafiador. Próximo à transição algumas grandezas físicas divergem, em especial o comprimento de correlação que é infinito. Uma forma de abordar o estudo é através do cálculo de expoentes críticos. Eles sinalizam como algumas grandezas termodinâmicas se comportam com a variação de parâmetros. Por exemplo, o comportamento do calor específico com a temperatura próxima à temperatura crítica. Neste trabalho investigamos o comportamento do calor específico próximo ao ponto crítico de sistemas físicos com interação de longo alcance. O sistema é descrito por um conjunto de partículas em um anel, que interagem pelo Hamiltoniano de Campo Médio. Utilizando a função de partição canônica obtemos o potencial de Helmholtz (F). A seguir, utilizando teoria de escala obtemos o expoente crítico associado ao calor específico. Os resultados estão em acordo com a literatura.

Palavras-chave: Termodinâmica, Longo Alcance, Expoente crítico 


\begin{abstract}
The phase transition study of thermodynamic systems near the critical point is challenging. Next to transition some physical quantities diverge, especially the correlation length that is infinite. One way to approach the study is through the calculating of critical exponents. They indicate how some thermodynamic quantities behave by varying parameters. For example, the specific heat behavior with the temperature close to the critical temperature. In this study, we investigate the specific heat behavior near the critical point of physical systems with long range interaction. The system is described by a set of particles in a ring, interacting by Hamiltonian Mean Field. Using the canonical partition function we obtain the Helmholtz potential (F). Furthermore, using scale theory we obtain the critical exponent associated with the specific heat. The results are in agreement with literature.
\end{abstract}

Keywords: Thermodynamics, Long-Range, Critical exponent 


\section{Lista de Figuras}

1.1 Diagrama de fases simples. . . . . . . . . . . . . . . . . . . . 4

1.2 Descontinuidades caracterizando transição de fase de $1^{\mathrm{a}}$ ordem (a) Descontinuidade na entropia, (b) Descontinuidade no volume. . . . . . . . . . . 6

1.3 Gráfico da entropia versus energia do sistema. Há duas regiões estáveis e uma região instável. . . . . . . . . . . . . . . . . . . . . . . .

1.4 Gráfico do potencial de Gibbs versus volume, a uma dada temperatura fixa

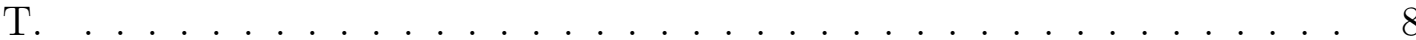

1.5 Potencial de Gibbs versus volume, para várias temperaturas $T_{1}<T_{2}<T_{3}$ $<T_{4}<T_{5} . T_{3}$ é a temperatura de transição. . . . . . . . . . . . 8

1.6 Potencial de Gibbs versus a pressão. . . . . . . . . . . . . . . . . . . . . . 8

1.7 Nesse sistema a diferença entre as densidades é o parâmetro de ordem. Ela vai diminuindo à medida que a temperatura se aproxima da temperatura crítica $\left(\mathrm{T}_{c}\right) \ldots \ldots \ldots \ldots \ldots \ldots \ldots \ldots$

1.8 Diagrama de fase de um ferromagneto simples. A linha de transição de fase

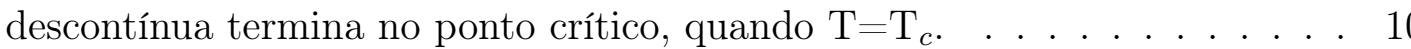

1.9 Magnetização versus temperatura, a linha vermelha representa a magnetização espontânea. . . . . . . . . . . . . . . . . . . . . . . . 11

2.1 Oito fluidos pertencentes à mesma classe de universalidade. . . . . . . . . . 16

2.2 Variação da energia livre de Landau pela magnetização para valores de (a) $a_{2}>0,(\mathrm{~b}) a_{2}=0,(\mathrm{c}) a_{2} \lesssim 0,(\mathrm{~d}) a_{2}<0 \ldots \ldots \ldots 17$

2.3 Bloco de sítios. . . . . . . . . . . . . . . . . . . . . 20

2.4 Bloco de sítios escalados. . . . . . . . . . . . . . . . . . . 20

3.1 Partícula localizada no centro de uma esfera com distribuição homogênea de partículas. O raio da esfera exterior é $R$ e da esfera interior é $\delta$. . . . . 23

3.2 Modelo de sistema com interação de curto alcance entre as partículas. . . . 25 
3.3 Modelo de sistema com interação de longo alcance entre as partículas. . 26

3.4 Possível representação do HMF . . . . . . . . . . . . . . . . . . . . 30

4.1 O gráfico da magnetização pela entropia, 4.1, nos mostra a existência de uma transição de fase contínua nesse sistema em $e=0,75$. . . . . . . . . 39

4.2 Curva calórica para o HMF microcanônico . . . . . . . . . . . . . . . . 39

4.3 Calor específico versus energia para o $\mathrm{HMF}$. . . . . . . . . . . . . . . . . 40

4.4 Gráficos com resolução da equação autoconsistente para diferentes valores de $\beta: 4,3.5,2.5$ e $1.5 \ldots \ldots \ldots \ldots \ldots 4 \ldots \ldots \ldots$

4.5 Gráfico de $m^{*}$ versus temperatura. . . . . . . . . . . . . . . . . . . 44

4.6 Curva calórica sistema HMF ensemble canônico. . . . . . . . . . . . . . . . 45

4.7 Calor específico versus temperatura para o $\mathrm{HMF} \ldots \ldots$. . . . . . . . . . 45 


\section{Lista de Símbolos}

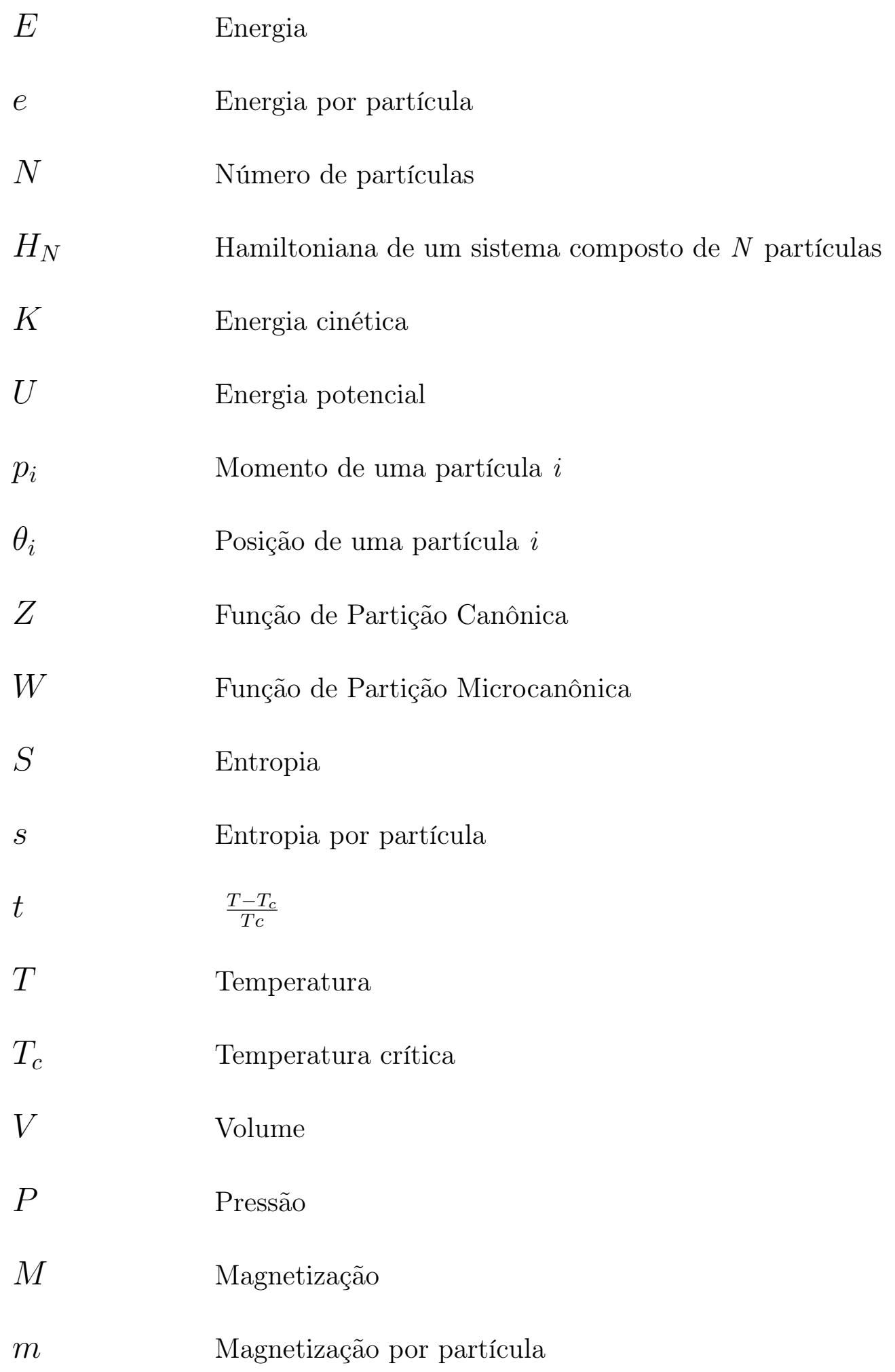




\begin{tabular}{|c|c|}
\hline$H$ & Campo Magnético \\
\hline$G$ & Potencial de Gibbs \\
\hline$g$ & Potencial de Gibbs por partícula \\
\hline$F$ & Energia Livre de Helmholtz \\
\hline$f$ & Energia Livre de Helmholtz por partícula \\
\hline$G_{\text {cor }}$ & Função de correlação \\
\hline$c_{x}$ & Calor Específico \\
\hline$\kappa_{x}$ & Compressibilidade \\
\hline$\chi_{x}$ & Susceptibilidade \\
\hline$k_{B}$ & Constante de Boltzmann \\
\hline$h$ & Constante de Plank \\
\hline$\beta$ & Inverso da temperatura, $\beta=\frac{1}{k_{B} T}$ \\
\hline$\omega$ & Densidade de probabilidade \\
\hline $\mathcal{L}^{-1}$ & Transformada inversa de Laplace \\
\hline$B_{i n v}$ & Função inversa de $\frac{I_{1}}{I_{0}}$ \\
\hline$L$ & Calor latente \\
\hline$\rho$ & Densidade do sistema \\
\hline$\rho_{c}$ & Densidade crítica \\
\hline
\end{tabular}




\section{Sumário}

Introdução 1

1 Transição de fase 4

1.1 Transição de fase descontínua (ou de primeira ordem) . . . . . . . . . . 5

1.2 Transição de fase contínua (ou de segunda ordem) . . . . . . . . . . . . 9

1.3 Transição de fase em termos da Energia de Helmholtz . . . . . . . . . . . . . 10

2 Expoentes críticos $\quad 13$

2.1 Definição . . . . . . . . . . . . . . . . . . . 13

2.2 Classe de universalidade . . . . . . . . . . . . . . . 15

2.3 Teoria de Landau e os expoentes críticos . . . . . . . . . . . . . . 16

2.4 Lei de Escala . . . . . . . . . . . . . . . . . . . . . . . . . . . . . . . 19

2.5 Cálculo dos expoentes críticos através da Teoria de Escala . . . . . . . . . 20

3 Sistemas físicos com interação de longo alcance 23

3.1 Definição . . . . . . . . . . . . . . . . . . . . . 23

3.1.1 A não aditividade da energia . . . . . . . . . . . . . . . . . . 24

3.1.2 A inequivalência entre os ensembles . . . . . . . . . . . 26

3.2 Teoria de Campo Médio . . . . . . . . . . . . . . . . . . . . . . . . . . . . 29

3.2.1 Hamiltoniano de Campo Médio (Hamiltonian Mean Field - HMF) 29

4 Estudo do Hamiltoniano de Campo Médio - HMF 32

4.1 Ensemble microcanônico . . . . . . . . . . . . . . . . . . . . . 32

4.2 Ensemble canônico . . . . . . . . . . . . . . . . . . . . . . . . . 40

5 Resultados e conclusões $\quad 47$

5.1 O cálculo do expoente crítico f . . . . . . . . . . . . . . 47

5.1.1 Energia de Hemlholtz com a primeira ordem das expansões . . . . . 48

5.1.2 Energia de Helmholtz com a segunda ordem das expansões . . . . . 50

5.2 Conclusões . . . . . . . . . . . . . . . . . . . . . . 52

Apêndice A Derivação da energia livre $\quad 54$ 
Referências Bibliográficas 


\section{Introdução}

Fase é um estado da matéria no qual as propriedades físicas da substância são uniformes em uma escala macroscópica [1]. Ela é caracterizada por uma função termodinâmica, por exemplo a energia livre de Helmholtz, e depende de alguns parâmetros macroscópicos, como a temperatura e o volume. Essa função descreve o estado do sistema e determina a fase da substância.

Exemplos familiares são as fases gasosa, líquida e sólida, mas existem também outros exemplos:

- Fases magnéticas: paramagnetismo, ferromagnetismo e antiferromagnetismo;

- Alguns metais apresentam uma fase supercondutora a baixas temperaturas;

- Cristais líquidos, cujas moléculas alongadas se alinham de modo a quebrar a simetria de rotação sem que haja quebra de simetria de translação;

- Fases diferentes aparecem também em outras áreas, tais como: economia e sociologia. Nessas áreas elas foram reconhecidas apenas recentemente e podemos estudar as transições entre as fases também [2] .

Estas fases possuem características que as tornam diferentes umas das outras. Em casos nos quais essa simetria das fases é distinta a transição entre elas é abrupta, já que há mudança de uma simetria para outra diferente. Essa transição é denominada descontínua. Ela pode ser vista como o resultado de uma falha no critério de estabilidade (convexidade ou concavidade) do potencial termodinâmico [3].

Além da transição de fase descontínua, outra transição muito estudada é a transição de fase contínua. Essa transição ocorre quando aproxima-se do ponto crítico. A transição de fase contínua explica muitos fenômenos como a opalescência crítica da água, na qual o material transparente ou translúcido adquire um aspecto ou tonalidade leitosa. Esse efeito deve-se ao comportamento da dispersão da luz no ponto crítico, dado que o comprimento de correlação entre os componentes do sistema tende ao infinito nesse ponto. 
Essa transição contínua tem sido foco de estudo na área de Física da Matéria Condensada principalmente pelo fato de que alguns sistemas distintos apresentam semelhanças de comportamento no ponto crítico. Além do fato de que há uma quebra de simetria que ainda não é completamente compreendida [4]. O estudo dessa transição traz muitos avanços práticos, como na área de supercondutividade e no estudo de líquidos super resfriados.

Nesse trabalho estudamos o comportamento de grandezas físicas de um sistema com interação de longo alcance próximo ao ponto crítico. Sistemas com interação de longo alcance são descritos por um potencial da forma:

$$
U(r)=\frac{1}{r^{\alpha}}
$$

sendo $\alpha$ um parâmetro que define o alcance da interação [5]. Para $\alpha<d$, com $d$ representando a dimensão física do sistema, o sistema possui interação de longo alcance.

Alguns sistemas com interação de longo alcance possuem uma característica peculiar em relação à maioria dos sistemas estudados em termodinâmica, que é a não aditividade da energia. Isso trás muitas consequências em uma abordagem termodinâmica, principalmente quanto à equivalência entre os ensembles. Portanto, esses sistemas são muito interessantes de estudar.

O primeiro capítulo apresenta uma revisão de conceitos de transição de fase e suas derivações. Apresentamos as diferenças entre transição de fase descontínua e contínua, as especificações de cada uma delas e como podem ser diferenciadas.

O segundo capítulo apresenta uma introdução sobre o conceito de expoente crítico, seu uso e significado. Apresenta, ainda, a teoria de Landau e como calcular os expoentes críticos a partir dela. Fazemos então uma breve discussão sobre a teoria de escala, sobre universalidade e classes.

O terceiro capítulo introduz a definição de sistemas com interação de longo alcance e inclui teoria de Campo Médio nessa categoria de sistemas (para $\alpha=0$ ), especificamente o Hamiltoniano de Campo Médio (HMF $\left.{ }^{1}\right)$.

Ao final do capítulo, calculamos a função de partição e a entropia do sistema no ensemble microcanônico. A seguir, calculamos a função de partição canônica e a energia de Helmholtz do sistema e, comparando esses dois sistemas, poderemos ver que são equivalentes.

\footnotetext{
${ }^{1}$ Sigla para a tradução em inglês Hamiltonian Mean Field
} 
Por fim, usando teoria de escala na função de energia de Helmholtz calculamos o valor do expoente crítico referente ao calor específico do sistema dado e comparamos com os resultados previstos pela teoria de Landau, sendo a primeira vez que esse expoente é calculado analiticamente para o sistema HMF pela teoria de escala. 


\section{Capítulo 1}

\section{Transição de fase}

Transições de fase são mudanças na fase de um sistema termodinâmico, causadas por variação de parâmetros macroscópicos que descrevem o sistema. Elas têm sido importantes há milênios como por exemplo no desenvolvimento de técnicas de fundição de metais para construção de ferramentas agrícolas, náuticas e militares o que culminou com o fim da Idade da Pedra. Atualmente a evaporação do petróleo destilado nos carburadores representa outro exemplo de notável aplicação prática das transições de fase. Entendê-las é uma das importantes tarefas dos físicos da Matéria Condensada [6].

Um diagrama de fases representa as fases de um sistema, dados alguns parâmetros macroscópicos, como mostra a figura (1.1). No diagrama de fases observamos regiões divididas por curvas de coexistência.

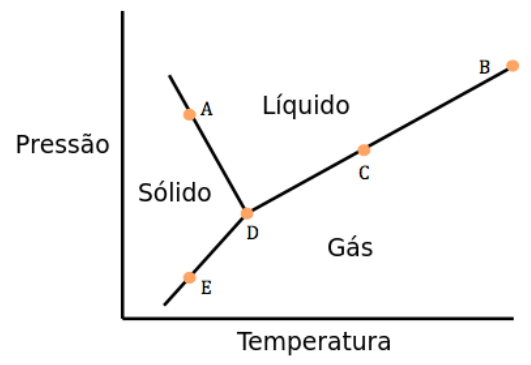

Figura 1.1: Diagrama de fases simples.

As curvas de coexistência são as curvas que separam as fases. Portanto, quando o sistema se encontra sobre uma dessas curvas, observamos a coexistência das duas fases. Por exemplo o ponto C, na figura (1.1), na curva liquido-gás onde as fases gasosa e líquida coexistem. Nesse ponto, a diferença entre as densidades do gás e do líquido é diferente de zero. E à medida que se fornece calor para o sistema, a temperatura do mesmo não muda (até que o sistema se encontre em apenas uma das fases). Esse calor cedido ao sistema 
na linha de coexistência é denominado calor latente. Atravessar essa curva significa ter mudado de fase.

O sistema será estável em uma região do diagrama de fases se a energia livre for mínima (como mostra a figura (1.4)) [7]. Por exemplo, para um sistema no estado gasoso a energia livre do gás terá um valor mínimo se comparada com as energias livres das outras duas fases (líquida e sólida).

O diagrama de fases apresenta dois pontos interessantes. O ponto triplo (ponto D na figura (1.1)), no qual as três fases coexistem. E o ponto crítico (ponto B na figura (1.1)), onde ocorre a transição de fase contínua. A região próxima ao ponto crítico é de difícil análise, contudo é possível estudá-la investigando-se como algumas grandezas físicas do sistema se comportam quando variamos, por exemplo, temperatura, volume ou campo magnético. Neste caso, pode-se prever o comportamento do sistema estudando os expoentes críticos.

\subsection{Transição de fase descontínua (ou de primeira or- dem)}

Intuitivamente esperamos que quando injetamos calor em um sistema, a pressão constante, a temperatura do mesmo aumente. Porém quando a temperatura atinge a "temperatura de derretimento"(no ponto A da figura (1.1)), por exemplo, a temperatura para de aumentar à medida que calor é injetado no sistema [3]. O calor adicional que está sendo inserido no sistema faz o material derreter, e o sistema não altera sua temperatura. A quantidade de material derretido no sistema vai depender da quantidade de calor fornecida ao sistema desde que a temperatura parou de aumentar (ou seja, ao chegar na curva de coexistência). Quando o material estiver todo derretido qualquer calor injetado no sistema fará, novamente, a temperatura do sistema aumentar.

Esse calor necessário para o derretimento do material é o calor latente. Por exemplo, o calor latente da transiçao água-gelo é $L=334 \mathrm{Jg}^{-1}$, que é a energia liberada quando as moléculas de $\mathrm{H}_{2} \mathrm{O}$ se arranjam em redes cristalinas hexagonais: acima do ponto de congelamento da água existe uma desordem no sistema, abaixo desse ponto existe uma ordem no sistema que agora está em forma de gelo [6]. Ou as moléculas estão em pacotes e em rede, na sua maioria, ou não estão em rede e não estão compactadas. À medida que a água vai sofrendo a transição, energia em forma de calor latente será liberada. 
A emissão, ou absorção, de calor latente nessa transição nos indica que a estrutura do material está sendo radicalmente reorientada [6]. Esse calor é essencial na descrição da transição de fase descontínua.

Outra característica dessa transição é uma mudança brusca em alguns parâmetros, como no volume e na entropia (figura 1.2).
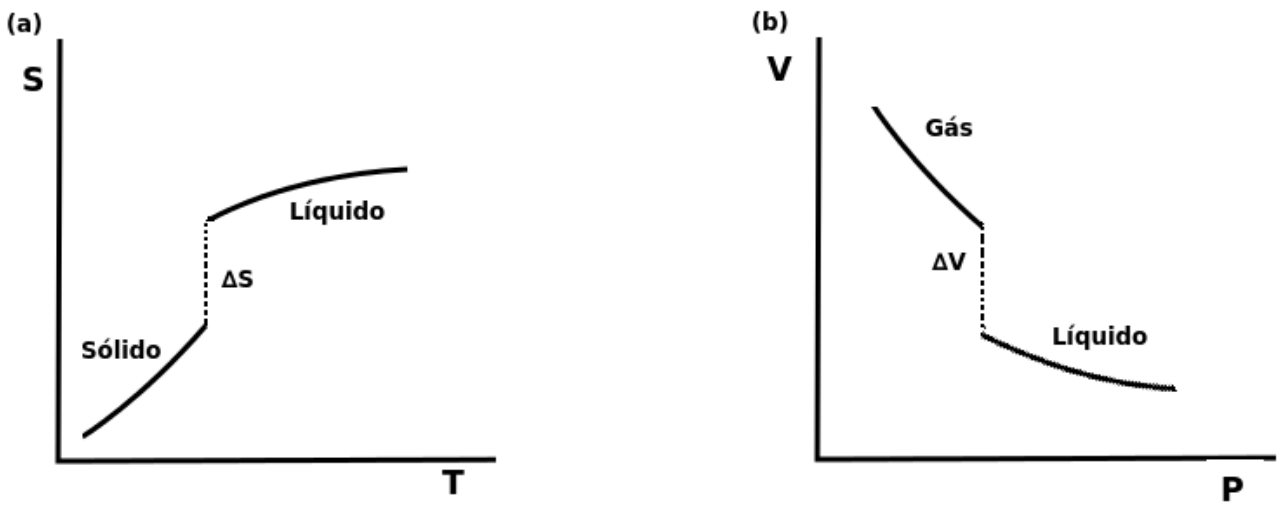

Figura 1.2: Descontinuidades caracterizando transição de fase de $1^{\mathrm{a}}$ ordem (a)Descontinuidade na entropia (b) Descontinuidade no volume.

Essa descontinuidade na entropia do sistema está diretamente ligada ao calor latente, pois o calor latente é definido como [3]:

$$
L=T \Delta S
$$

em que T é a temperatura na qual começa a transição e $\Delta S$ é a diferença entre a entropia da fase para qual o sistema está transitando e a entropia da fase na qual o sistema se encontra. Na linha de coexistência da transição de fase descontínua a entropia de uma fase é diferente da outra, $\log \Delta S \neq 0$ e existe calor latente.

Outra forma de observar a transição de fase descontínua é pela análise do potencial de Gibbs do sistema. O potencial de Gibbs é uma função termodinâmica que depende das grandezas termodinâmicas, temperatura $(\mathrm{T})$, pressão $(\mathrm{P})$ e número de partículas $(\mathrm{N})$.

Na figura (1.3) notamos duas regiões de estabilidade e uma região de instabilidade. Quando o sistema se encontra na região de instabilidade, qualquer perturbação faz o sistema ir para alguma das regiões de estabilidade. As duas regiões de estabilidade representam duas fases diferentes e passar de uma para a outra é o que chamamos de mudança de fase.

As instabilidades estão associadas às flutuações das variáveis termodinâmicas no sistema, logo a transição de fase está associada às flutuações. Ao sair de uma região de 


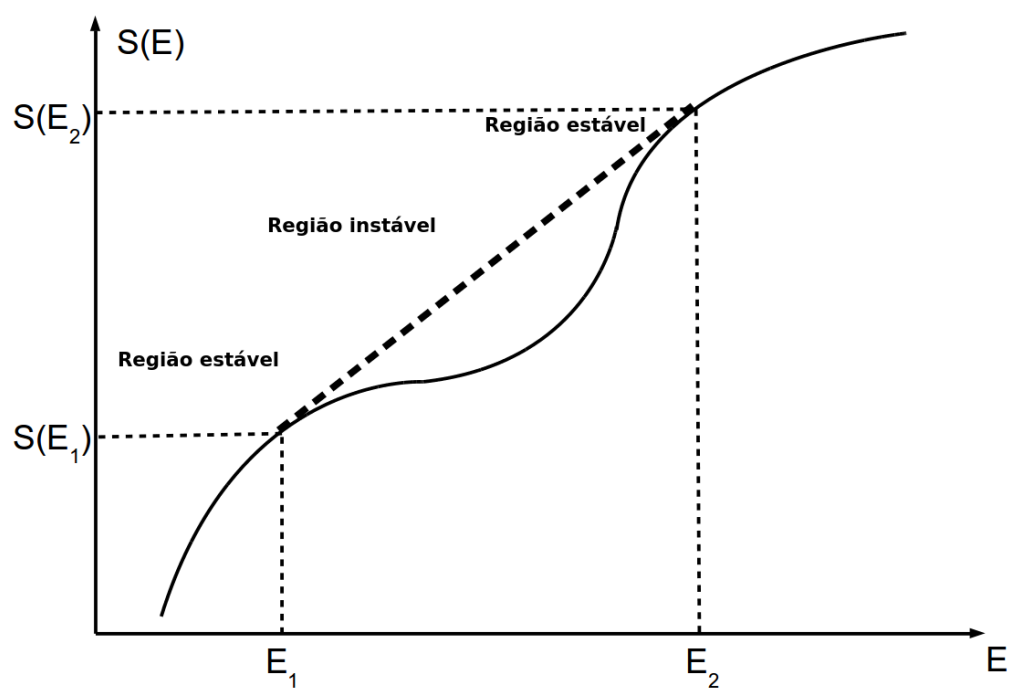

Figura 1.3: Gráfico da entropia versus energia do sistema. Há duas regiões estáveis e uma região instável (Imagem retirada de [8]).

equilíbrio (devido ao aumento do volume, por exemplo) as flutuações aumentam muito na região instável e depois o sistema passa para a próxima região estável e com poucas flutuações.

No equilíbrio o sistema está estável no mínimo valor do potencial de Gibbs [3],então a primeira derivada do potencial de Gibbs pelo volume é nula e a segunda derivada é positiva ou nula:

$$
\left(\frac{\partial G}{\partial V}\right)_{T}=0
$$

e

$$
\left(\frac{\partial^{2} G}{\partial V^{2}}\right)_{T} \geq 0
$$

Dado o potencial de Gibbs a uma temperatura constante T, o gráfico de $G$ por $V$, volume, é representado na figura (1.4). O ponto vermelho na figura (1.4) mostra a fase de maior estabilidade, então é nessa fase que o sistema se encontra nessa temperatura. Cada curva convexa representa uma fase e a curva côncava é a região de instabilidade.

O gráfico do potencial de Gibbs pelo volume, para várias temperaturas diferentes, é dado na figura (1.5). Para $T_{1}$ e $T_{2}$ o estado mais estável é o do lado esquerdo da figura. Em $T_{3}$ há coexistência de fases e em $T_{4}$ e $T_{5}$ o sistema já mudou de fase e agora o estado mais estável é do lado direito.

Na figura (1.6) podemos notar que o ponto vermelho é o ponto de coexistência, pois 


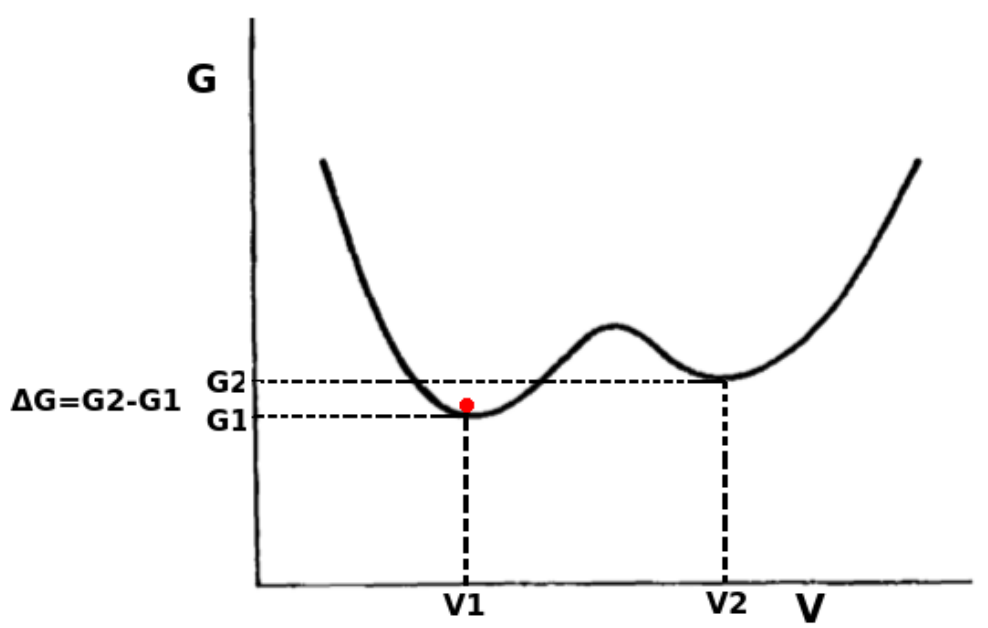

Figura 1.4: Gráfico do potencial de Gibbs versus volume, a uma dada temperatura fixa T.

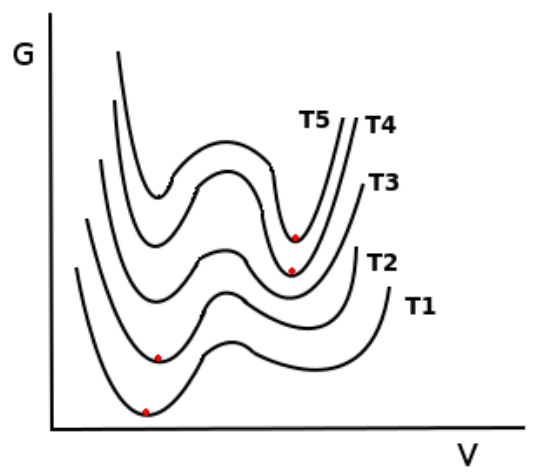

Figura 1.5: Potencial de Gibbs versus volume, para várias temperaturas $T_{1}<T_{2}<T_{3}<$ $T_{4}<T_{5} . T_{3}$ é a temperatura de transição.

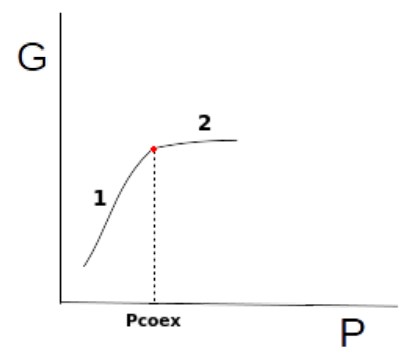

Figura 1.6: Potencial de Gibbs versus a pressão. 
a derivada do potencial de Gibbs pela pressão (no ponto vermelho) nos dá o volume:

$$
\left.\left(\frac{\partial G_{1}}{\partial P_{\text {coex }}}\right)_{T}=V_{1} \text { (na região } 1\right) \text { e }\left(\frac{\partial G_{2}}{\partial P_{\text {coex }}}\right)_{T}=V_{2} \text { (na região 2). Ou seja, o volume }
$$

é descontínuo nesse ponto (a primeira derivada do potencial é descontínua) o que está associado a uma transição de fase.

\subsection{Transição de fase contínua (ou de segunda ordem)}

Além da transição de fase descontínua (a mais comum), existe a transição de fase contínua (ou transição de fase de segunda ordem). Ela ocorre quando o sistema atinge o ponto crítico. Nesse ponto o sistema sofre uma transição contínua que é caracterizada pela impossibilidade de se distinguir a fase na qual o sistema se encontra.

Uma substância na fase líquida pode mudar para a fase gasosa, por exemplo, contornando o ponto crítico, de forma contínua e não brusca. Ou seja, sem precisar passar pela linha de transição.

Não observamos calor latente nessa transição (como obervamos na descontínua), e também não observamos descontinuidade no volume e na entropia. Por outro lado, notamos que o comprimento de correlação diverge e que as flutuações são muito relevantes no ponto crítico.

A fase de um sistema pode, também, ser caracterizada por um parâmetro de ordem. O parâmetro de ordem indica se elementos microscópicos que compõem a fase macroscópica estão ordenados ou em algum estado similar [1]. Ele é associado à simetria do sistema e mede o grau de assimetria de um sistema quando ocorre uma quebra da simetria natural [1]. No contexto de Landau, na fase ordenada (estado de baixa simetria) o parâmetro de ordem é diferente de zero e na fase desordenada (estado simétrico) o parâmetro de ordem é zero.

A diferença entre as densidades de um gás e um líquido é um exemplo de parâmetro de ordem, como mostra a figura (1.7). Em baixas temperaturas essa diferença é não nula, mas quando o sistema vai se aproximando do ponto crítico essa diferença vai tendendo a zero [9].

Em uma transição de fase contínua, a simetria do sistema é quebrada e podemos, então, atestar a existência dessa transição através da análise do parâmetro de ordem do sistema. Em um sistema magnético, por exemplo, a magnetização espontânea é o parâmetro de ordem, que mede a assimetria nos estados do sistema [1].

O fato da curva que delimita as fases terminar no ponto crítico significa que pode-se 


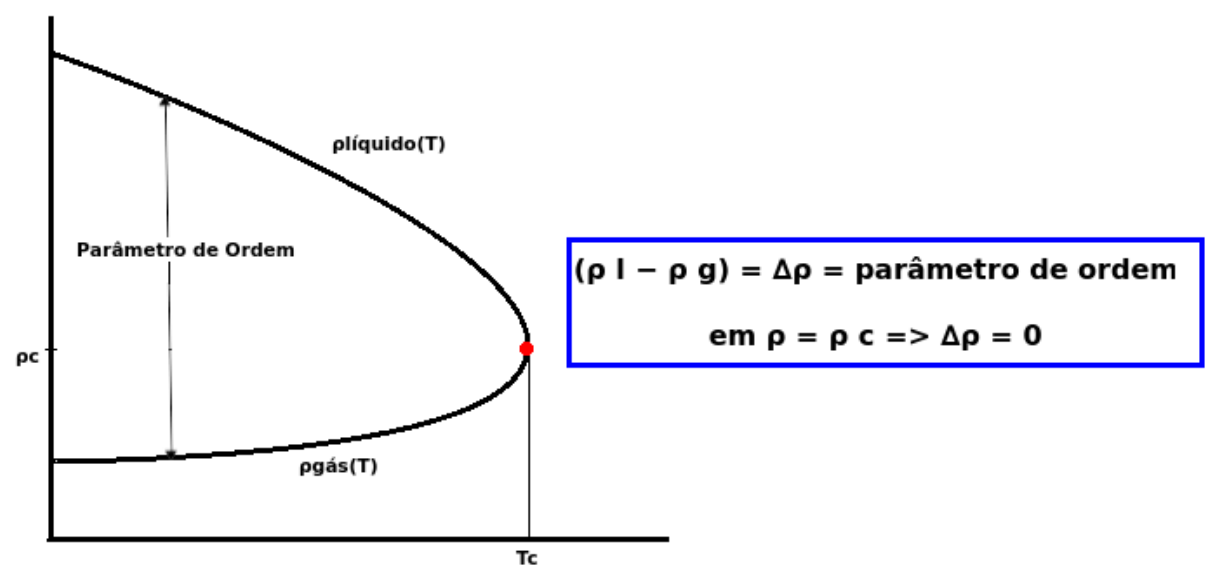

Figura 1.7: Nesse sistema a diferença entre as densidades é o parâmetro de ordem. Ela vai diminuindo à medida que a temperatura se aproxima da temperatura crítica $\left(\mathrm{T}_{c}\right)$.

passar da fase ferromagnética para a fase paramagnética, por exemplo, (ou da fase líquida para a gasosa) de forma contínua, como mostrado na figura (1.8)[9].

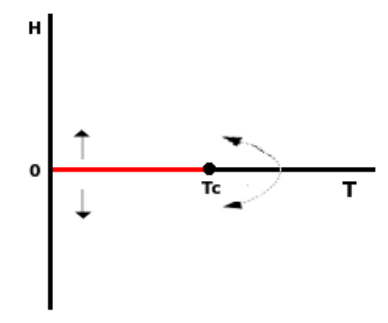

Figura 1.8: Diagrama de fase de um ferromagneto simples. A linha de transição de fase descontínua termina no ponto crítico, quando $\mathrm{T}=\mathrm{T}_{c}$.

Em um sistema ferromagnético, sem campo externo, à medida que aumentamos a temperatura do sistema a magnetização espontânea vai diminuindo até que alcançamos uma certa temperatura crítica e a magnetização vai a zero (figura (1.9)). Há uma quebra de simetria nesse momento, o que caracteriza uma transição de fase contínua.

\subsection{Transição de fase em termos da Energia de Helmholtz}

A caracterização de uma transição de fase pode ser dada pela presença de descontinuidades nos potenciais termodinâmicos ou suas derivadas.

A energia livre de Helmholtz, $\mathrm{F}(\mathrm{T}, \mathrm{V}, \mathrm{N})$, é uma grandeza dependente da temperatura, do volume e do número de partículas. Nem toda a energia interna de um sistema é passível de produzir trabalho visto que uma parcela desta energia encontra-se diretamente associada à entropia. Portanto, $F$ mede a parcela de energia interna de um sistema possível de ser utilizada em forma de trabalho. Pode ser calculada através da transformada 


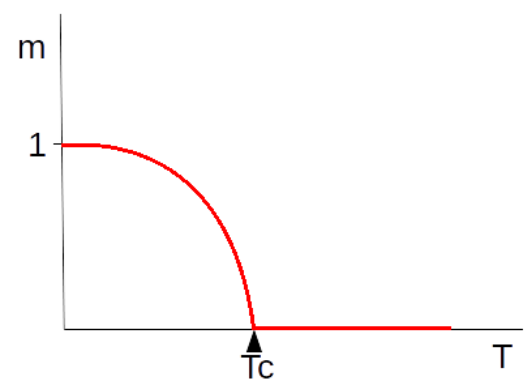

Figura 1.9: Magnetização versus temperatura, a linha vermelha representa a magnetização espontânea.

de Legendre da energia:

$$
F(T, V, N)=E-T S
$$

onde $E$ é a energia interna, $T$ é a temperatura e $S$ a entropia do sistema.

Podemos classificar as transições de fase de acordo com a ordem das derivadas da energia livre do sistema. Ehrenfest classificou as transições de fases como sendo de ordem N, caso a N-ésima derivada da energia livre, no ponto de transição, apresentasse uma descontinuidade.

Como exemplo, quando as primeiras derivadas da energia de Helmholtz (que nos dão a magnetização e a entropia, por exemplo) são descontínuas no ponto de transição, dizemos que a transiçao é de primeira ordem:

$$
\begin{aligned}
& P=-\left(\frac{\partial f}{\partial v}\right)_{T} ; \\
& m=-\left(\frac{\partial f}{\partial H}\right)_{T} ; \\
& s=-\left(\frac{\partial f}{\partial T}\right)_{H} ;
\end{aligned}
$$

sendo $P$ a pressão do sistema, $v$ o volume do sistema, $m$ a magnetização do sistema, $s$ entropia do sistema, $H$ o campo externo e $f$ a energia de Helmholtz por partícula.

Já as transições de fase contínuas são caracterizadas pela divergência ou descontinuidade nas segundas derivadas da energia livre na região crítica. Apesar de algumas serem apenas divergências, e não descontinuidades (motivo pelo qual a nomenclatura de Ehrenfest não se adequa mais), ela é conhecida como transição de segunda ordem. Como exemplo, as segundas derivadas da energia livre de Helmholtz, que nos dão o calor específico $\left(c_{x}\right.$, ou seja o calor específico com relação a uma constante $\left.x\right)$ e a susceptibilidade 
$(\chi):$

$$
\begin{gathered}
\chi_{T}=\left(-\frac{\partial^{2} f}{\partial H^{2}}\right)_{T} \\
c_{x}=-T_{c}\left(\frac{\partial^{2} f}{\partial T^{2}}\right)_{x} .
\end{gathered}
$$

A transição contínua será nosso foco nesse trabalho. Neste caso devemos obter o potencial termodinâmico para posteriormente obter o expoente crítico. Este estudo será realizado nos capítulos 4 e 5 deste trabalho. 


\section{Capítulo 2}

\section{Expoentes críticos}

Um dos problemas existentes ao se estudar transição de fase contínua é saber o que acontece com o sistema na região próxima ao ponto crítico, pois, no ponto crítico, algumas grandezas do sistema possuem singularidades. Por exemplo, a susceptibilidade, o calor específico são descontínuos, o comprimento de correlação entre as partículas diverge e as flutuações dominam em relação às médias.

É muito importante a compreensão de como essas grandezas divergem. Podemos expressar esse comportamento atípico em função de potências. Essas potências são chamadas expoentes críticos, que descrevem o comportamento do sistema próximo ao ponto crítico.

\subsection{Definição}

O expoente crítico associado à função $F(t)$ é definido da seguinte forma [10]

$$
\lambda=\lim _{t \rightarrow 0} \frac{\ln |F(t)|}{\ln |t|}
$$

ou mais usualmente como:

$$
F(t) \sim|t|^{\lambda}
$$

Sendo,

$$
t=\frac{\left(T-T_{c}\right)}{T_{c}}
$$

onde $T_{c}$ é a temperatura crítica do sistema.

E são usualmente escritos, em um sistema magnético, como:

- O expoente $\alpha$ descreve o comportamento do calor específico $\left(c_{H}\right)$ na região crítica, 
em função de t, quando o campo magnético $H=0$;

$$
c_{H} \sim t^{-\alpha}
$$

- O expoente $\beta$ descreve o comportamento do parâmetro de ordem $(m)$ do sistema, em função de t, quando o campo magnético $H=0$, na região crítica;

$$
m \sim t^{\beta}
$$

- O expoente $\gamma$ descreve a suceptibilidade $(\chi)$ do sistema na região crítica, em função de t, quando o campo magnético $H=0$;

$$
\chi_{H} \sim t^{-\gamma}
$$

- O expoente $\delta$ descreve o comportamento do parâmetro de ordem $(m)$ do sistema, em função do campo magnético, quando $T=T_{c}$ e o campo magnético $H \rightarrow 0$;

$$
m \sim H^{\frac{1}{\delta}}
$$

- O expoente $\eta$ descreve a função de correlação $\left(G_{c o r}\right)$ na região crítica;

$$
G_{c o r} \sim \frac{1}{r^{d-2+\eta}}
$$

onde $r$ é a distância espacial e $d$ é a dimensão física do sistema.

- O expoente $\nu$ descreve o comprimento de correlação $(\xi)$ na região crítica, em função de t;

$$
\xi \sim t^{-\nu}
$$

Os expoentes críticos obedecem às chamadas leis de escala, que descrevem a relação entre dois ou mais expoentes [6]. Essas leis de escala são consequência da invariância por escala da energia livre, como mostrado na seção (2.4), e mostram o vínculo que existe entre os expoentes [6]. Observando as leis de escala, com 2 (ou 3 no caso de (2.8)) expoentes críticos pode-se achar o terceiro.

Os expoentes críticos relacionam-se entre si de acordo com as seguintes leis: 
- Lei de Rushbrooke

$$
2 \beta+\gamma=2-\alpha
$$

- Lei de Griffiths

$$
2 \beta \delta-\gamma=2-\alpha
$$

- Lei de Fisher

$$
\gamma=\nu(2-\eta)
$$

- Lei de Josephson

$$
\nu d=2-\alpha,
$$

\subsection{Classe de universalidade}

Outro aspecto interessante do fenômeno crítico é a semelhança do comportamento de sistemas diferentes quando estão próximos ao ponto crítico. O valor numérico dos expoentes críticos é idêntico em grandes grupos de sistemas físicos diferentes [4].

Enquanto a temperatura crítica de cada sistema depende de detalhes como interações interatômicas, os expoentes críticos dependem apenas de alguns parâmetros fundamentais como a dimensionalidade do sistema e o número de componentes do parâmetro de ordem [10].

A figura (2.1) mostra a coexistência de 8 curvas de fluidos diferentes. Próximo ao ponto crítico (e longe também) os dados se encontram sobre a mesma curva. O expoente crítico que descreve o comportamento do parâmetro de ordem desses sistemas $(\Delta \rho)$ :

$$
\Delta \rho \sim t^{\beta}
$$

é $\beta=\frac{1}{3}$, para todos os 8 fluidos [6].

Isso é chamado de comportamento universal. Esse comportamento está ligado apenas à dimensionalidade, número de componentes do parâmetro de ordem do sistema e se as interações são de longo ou curto alcance [12].

Ou seja, apesar de outras tantas diferenças entre alguns sistemas eles podem ser descritos pelos mesmos parâmetros na região crítica, os expoentes críticos. Dois sistemas com os mesmos valores de expoentes críticos pertencem à mesma classe de universalidade.

Se os sistemas pertencem à mesma classe de universalidade é possível essa comparação 


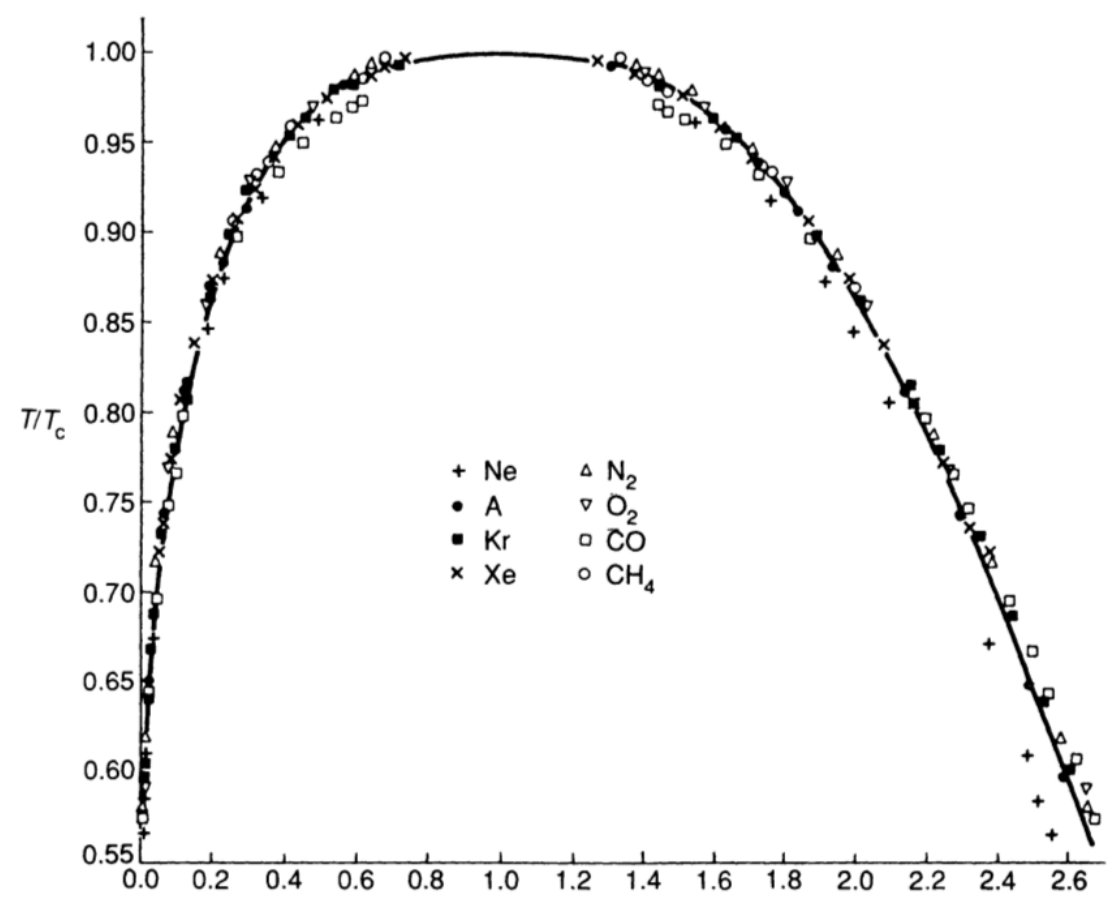

Figura 2.1: Oito fluidos pertencentes à mesma classe de universalidade. (Imagem retirada do artigo [11].

na região crítica, tendo a certeza de que os modelos possuem a dimensão certa e que a simetria do parâmetro de ordem está corretamente descrita [10].

Por exemplo, sistemas estudados a partir da teoria de campo médio têm valores de expoentes críticos clássicos:

Tabela 2.1: Expoentes críticos clássicos para sistemas de campo médio

\begin{tabular}{|l|l|l|l|l|}
\hline Expoente & $\beta$ & $\gamma$ & $\delta$ & $\alpha$ \\
\hline Valor & $\frac{1}{2}$ & 1 & 3 & 0 \\
\hline
\end{tabular}

\subsection{Teoria de Landau e os expoentes críticos}

A Teoria de Landau não só prediz transição de fase como demonstra de forma muito clara como os expoentes críticos dependem da simetria do parâmetro de ordem. Landau sugeriu a introdução de um parâmetro extra na energia livre, $m$, que é chamado de parâmetro de ordem, e é usado para distinguir a fase do sistema.

Ele se baseou em uma descrição das transições a partir da simetria das fases do sistema [7]. Então, agora, a energia livre do sistema seria escrita em função de três parâmetros $F(V, T, m)$. Para altas temperaturas $T>T_{c}, m=0$ e isso indica uma desordem e simetria no estado e para temperaturas menores que $T_{c}, m \neq 0$ indicando um estado ordenado e assimétrico. 
Landau assumiu que a energia livre do sistema é minimizada pelo parâmetro de ordem $\left(\frac{\partial F}{\partial m}\right)_{T}=0$ e pode ser expandida como uma série de potências do parâmetro de ordem $m$ [2], pois próximo ao ponto crítico $m \approx 0$. Por exemplo, para um sistema ferromagneto na ausência de campo externo:

$$
F=F_{0}+a_{1} m+a_{2} m^{2}+a_{3} m^{3}+a_{4} m^{4}+\ldots
$$

sendo, $a_{i}=\left(\frac{\partial^{i} F}{\partial m^{i}}\right)_{T}, \operatorname{com} i=1, \ldots, n$.

Para um sistema magnético e identificando a magnatização como parâmetro de ordem, apenas os termos pares são invariantes quanto a inversão do sinal da magnetização, $\operatorname{logo} a_{3}=0$. E a primeira derivada de $F$ em relação a $m$ é nula, dado que a energia livre é minimizada no equilíbrio, em relação ao parâmetro de ordem. Portanto podemos reescrever a equação (2.11) da seguinte forma:

$$
F=F_{0}+a_{2} m^{2}+a_{4} m^{4}
$$

A série vai apenas até termos de ordem 4, pois se $a_{4}$ é positivo os termos que vem depois não interferem no comportamento crítico do sistema [10].

Nos gráficos da figura (2.2) podemos observar o comportamento da energia livre de Landau em função de $m$.

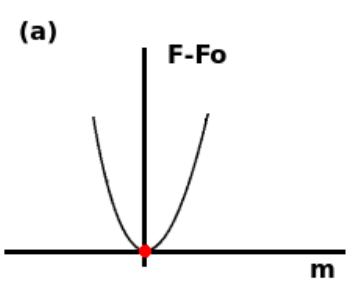

(c)

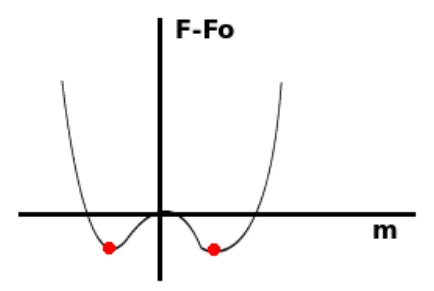

(b)

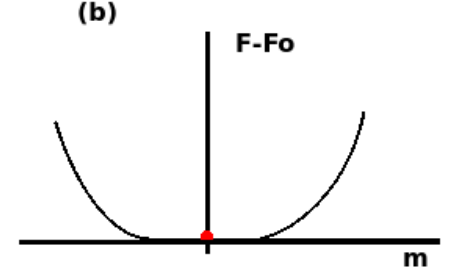

(d)

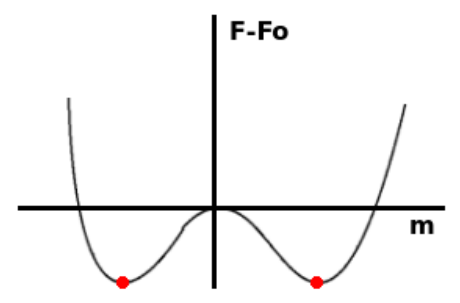

Figura 2.2: Variação da energia livre de Landau pela magnetização para valores de (a) $a_{2}>0$, (b) $a_{2}=0$, (c) $a_{2} \lesssim 0$, (d) $a_{2}<0$.

A partir da figura (2.2), observamos que o comportamento de $F$ pode ser descrito por: (i) $a_{2}>0$ o valor mínimo de $F$ é no ponto $m=0$ mostrando que o sistema está na 
fase paramagnética, (ii) $a_{2}=0$ corresponde a temperatura crítica, onde a magnetização espontânea aparece e (iii) $a_{2}<0$ o mínimo estável da energia livre corresponde a valores diferentes de zero, mostrando que o sistema está na fase ferromagnética [10].

Portanto, pela figura (2.2) vemos que $a_{2}$ tem uma correspondência com a temperatura, pois $m$ varia com a temperatura, e podemos reescrever:

$$
a_{2}=\tilde{a}_{2} t
$$

sendo

$$
t=\frac{\left(T-T_{c}\right)}{T_{c}}
$$

Observando o formato da curva da energia livre, na figura (2.2), à medida que $a_{2}$ decresce podemos notar que a magnetização torna-se não-zero de forma contínua, como podemos observar na figura (1.9), o que caracteriza uma transição de fases contínua.

É possível calcular os expoentes críticos pela Teoria de Landau. A derivada da equação (2.12) com respeito a $m$ é:

$$
\left(\frac{\partial F}{\partial m}\right)_{T}=2 \tilde{a}_{2} t m+4 a_{4} m^{3}=0
$$

e tem como solução estável, para

- (i) $t<0$, ou seja, $T<T_{c}$ :

$$
m \sim(-t)^{\frac{1}{2}}
$$

- (ii) $t>0$, ou seja, $T>T_{c}$ :

$$
m=0 .
$$

Portanto o valor do expoente crítico referente ao parâmetro de ordem $(m)$ descrito na equação (2.2) é $\beta=\frac{1}{2}$.

O expoente crítico referente ao calor específico, $c_{x}=-T\left(\frac{\partial^{2} F}{\partial T^{2}}\right)_{x}$, pode ser calculado usando a equação (2.13) para reescrever a equação (2.12) em termos de $t$ :

$$
F=F_{0}-\frac{\tilde{a}_{2}^{2} t^{2}}{4 a_{4}}+O\left(t^{3}\right)
$$


para $t<0 . \mathrm{E}$

$$
F=F_{0}
$$

para $t>0$.

Sendo o calor específico correspondente à segunda derivada da energia livre (segunda derivada da equação (2.16) ou da equação (2.17), dependendo do valor de $t$ ) em relação à temperatura notamos que para $t<0$ ele tende a um valor constante e para $t>0$ ele é nulo. Logo existe uma descontinuidade no calor específico na temperatura crítica e o expoente crítico referente ao calor específico é nulo ( $\alpha=0$, equação 2.1$)$, pois não depende da temperatura.

\subsection{Lei de Escala}

Teoria de escala é um método muito usado para descrever sistemas de forma mais simples. Em sistemas físicos é muito usada para construir parâmetros adimensionais. Considere uma função homogênea $f(x, y)$ de ordem $d$, podemos usar teoria de escala e a definir como:

$$
f(\lambda x, \lambda y)=\lambda^{d} f(x, y)
$$

escolhendo $\lambda=\frac{1}{x}$, podemos reescrever (2.18):

$$
f(x, y)=x^{d} f\left(1, \frac{y}{x}\right)=x^{d} \psi\left(\frac{y}{x}\right)
$$

A equação (2.19) mostra que uma função homogênea de duas variáveis pode ser escalada como uma função de uma única variável. Dessa forma, todos os sistemas descritos por essa função colapsam para uma mesma curva [2].

Podemos, também, considerar uma função homogênea generalizada:

$$
f\left(\lambda^{a} x, \lambda^{b} y\right)=\lambda^{d} f(x, y)
$$

nos casos em que $a=b$ a função se reduz a uma funcão homogênea normal.

O objetivo dessa técnica é diminuir os graus de liberdade do sistema estudado. Mesmo que não se saiba a solução para o problema com duas variáveis, nem a solução do problema com apenas uma variável, a comparação entre os dois pode nos dar alguma informação que nos ajude a resolver o problema. Usaremos esse método no capítulo 5 para obter o 
expoente crítico $\alpha$.

Considere um sítio como mostra a figura (2.3). Esse sítio pode ser escalado em um número menor de sítios, a cada conjunto de quatro pontos (pretos), formamos apenas um bloco (vermelho), como mostra a figura (2.4), que representa o conjunto de 4 pontos (pretos).

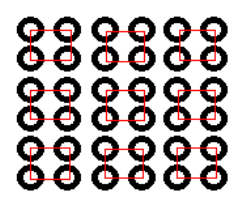

Figura 2.3: Bloco de sítios.

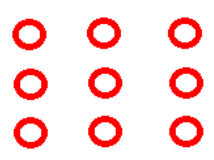

Figura 2.4: Bloco de sítios escalados.

Kadanoff propôs que dado $T$, a temperatura de cada ponto (preto), e $T^{\prime}$ a temperatura de cada bloco (vermelho), e o mesmo para um campo externo $H$ e $H^{\prime}$, do qual o sistema depende, podemos escrever:

$$
\begin{aligned}
& T^{\prime}=L^{x} T, \\
& H^{\prime}=L^{y} H .
\end{aligned}
$$

Portanto, considerando a energia livre do sistema com os pontos (pretos) $f(T, H)$ e a energia livre com os blocos (vermelhos) $f\left(T^{\prime}, H^{\prime}\right)$, e sabendo que a energia livre do bloco é a soma das energias livres dos pontos que o compõem, podemos escrever, em $d$ dimensões:

$$
L^{d} f(T, H)=f\left(T^{\prime}, H^{\prime}\right)=f\left(L^{x} T, L^{y} H\right)
$$

\subsection{Cálculo dos expoentes críticos através da Teoria de Escala}

Para um sistema magnético, próximo à $T_{c}$, a energia livre por partícula $f$ pode ser aproximada, usando teoria de escala e sabendo que $\lambda$ pode ser um valor qualquer: 


$$
\lambda f(t, H)=f\left(\lambda^{z} t, \lambda^{x} H\right)
$$

fazendo $\lambda=t^{-\frac{1}{z}}$, temos

$$
f(t, H)=t^{\frac{1}{z}} f\left(\frac{H}{t^{\frac{x}{z}}}\right) .
$$

A equação (2.22) é chamada de teste de escala de Widom. Vamos usar a equação de Widom para calcular os expoentes críticos.

A partir da equação (2.22), o parâmetro de ordem de um sistema, por exemplo a magnetização, pode ser calculado da seguinte forma:

$$
\begin{aligned}
m_{H=0} & =-\left(\frac{\partial f}{\partial H}\right)_{T} \\
& =-\left(\frac{t^{\frac{1}{z}}}{t^{\frac{x}{z}}}\right) f^{\prime}(0) .
\end{aligned}
$$

Então, dada a equação (2.2) que nos mostra o comportamento do parâmetro de ordem em relação a $t$, sabemos que $\beta=\frac{1-x}{z}$.

A susceptibilidade pode ser calculada pela segunda derivada da energia livre em relação a $H$ :

$$
\begin{aligned}
\chi_{H=0} & =-\left(\frac{\partial^{2} f}{\partial H^{2}}\right)_{T} \\
& =t^{\frac{(1-2 x)}{z}} f^{\prime \prime}(0) .
\end{aligned}
$$

Então, dada a equação (2.3) que nos mostra o comportamento da susceptibilidade em relação a $t$, sabemos que $\gamma=\frac{2 x-1}{z}$.

O calor específico será calculado pela segunda derivada da energia livre em relação a temperatura, para $H=0$ :

$$
\begin{aligned}
c_{H} & =-T_{c}\left(\frac{\partial^{2} f}{\partial T^{2}}\right)_{H} \\
& =-\frac{1}{T c} \frac{\partial^{2}}{\partial t^{2}}\left[t^{\frac{1}{z}} f\left(\frac{H}{t^{\frac{x}{z}}}\right)\right] \\
& =-\frac{f(0)}{T c} \frac{1}{z}\left(\frac{1}{z}-1\right) t^{\left(\frac{1}{z}-2\right)} .
\end{aligned}
$$

Então, dada a equação (2.1), que nos mostra o comportamento do calor específico em 
relação a $t$, sabemos que $\alpha=2-\frac{1}{z}$.

Podemos derivar as leis de escala (2.7), (2.8), (2.9) e (2.10) resolvendo as equações achadas para (2.23), (2.24) e (2.25), isolando $x$ e $z$ e achando as relações entre os expoentes.

Vamos usar essa aplicação da lei de escala para calcular o expoente crítico clássico $\alpha$ do sistema estudado. 


\section{Capítulo 3}

\section{Sistemas físicos com interação de longo}

\section{alcance}

\subsection{Definição}

Uma interação de longo alcance é descrita por um potencial entre partículas da forma:

$$
U(r)=\frac{1}{r^{\alpha}}
$$

sendo $\alpha$ o parâmetro que define o alcance da interação e $\alpha<d$, com $d$ representando a dimensão física do sistema [5].

Vamos excluir a contribuição de partículas localizadas a curtas distâncias $(r<\delta)$, figura (3.1), uma vez que o interesse é a natureza de longo alcance do potencial e para evitar divergência a curtas distâncias.

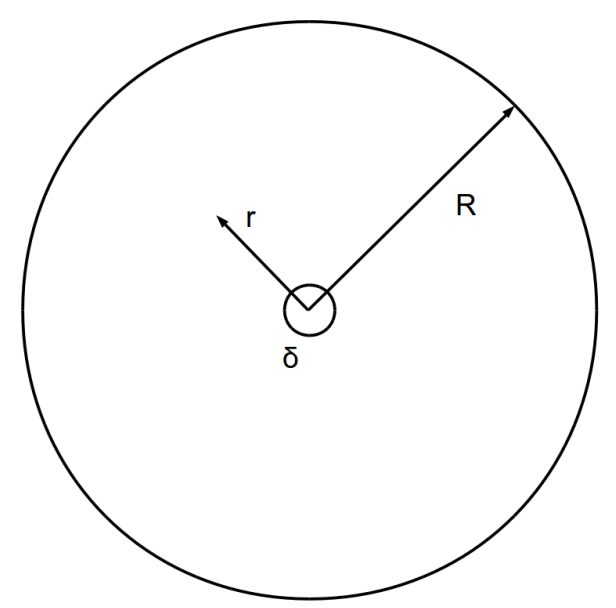

Figura 3.1: Partícula localizada no centro de uma esfera com distribuição homogênea de partículas. O raio da esfera exterior é $R$ e da esfera interior é $\delta$. 
Modelos de campo médio, como o Hamiltoniano de Campo Médio, possuem valor de $\alpha=0$, ou seja, a interação entre as partículas não depende da distância entre elas [5]. E sendo $\alpha=0<d$, sistemas de campo médio possuem interação de longo alcance.

A energia potencial $U$ do sistema é dada por [8]:

$$
\begin{gathered}
U=\int_{\delta}^{R} \rho \frac{1}{r^{\alpha}} d^{d} \vec{r}= \\
=\rho \Omega_{d} \int_{\delta}^{R} r^{d-\alpha-1} d^{d} r= \\
=\left.\frac{\rho \Omega_{d}}{d-\alpha} r^{d-\alpha}\right|_{\delta} ^{R} \\
U=\frac{\rho \Omega_{d}}{d-\alpha}\left[R^{d-\alpha}-\delta^{d-\alpha}\right], \quad \text { se } \alpha \neq d
\end{gathered}
$$

sendo $\rho$ a densidade e $\Omega_{d}$ o volume angular em $d$ dimensões.

$$
U=\left\{\begin{array}{cc}
\frac{\rho \Omega_{d}}{d-\alpha}\left[R^{d-\alpha}-\delta^{d-\alpha}\right], & \alpha \neq d . \\
\rho \Omega_{d} \ln \left(\frac{R}{\delta}\right), & \alpha=d .
\end{array}\right.
$$

De (3.3), se $\alpha>d$ então a energia potencial total do sistema é finita quando $R \rightarrow$ $\infty$, neste caso a energia cresce linearmente com o tamanho do sistema e dizemos que a interação é de curto alcance. Se $\alpha \leq d$ a energia potencial total do sistema diverge quando $R \rightarrow \infty$, ou seja, a energia cresce superlinearmente com o tamanho do sistema e a interação é dita de longo alcance [8].

\subsubsection{A não aditividade da energia}

A energia de um sistema é dita aditiva quando somamos as energias de cada subsistema que o compõe e o total é igual à energia do sistema. Por exemplo, dado um sistema composto por 2 subsistemas, a soma das energias $E_{1}+E_{2}$ tem que ser igual a $E$, a energia total do sistema.

A aditividade da energia em um sistema termodinâmico é observada em sistemas com interação de curto alcance, e é muito importante para garantir a equivalência entre os ensembles. Vamos considerar um sistema dividido em dois subsistemas iguais, em contato e isolados do resto do universo. O vínculo permite a troca de energia entre os dois subsistemas, mas não permite troca de partículas. A energia total do sistema sempre 
se mantém constante e pode ser calculada

$$
E_{1}+E_{2}+E_{12}=E
$$

onde $E_{1}$ é a energia de interação entre as partículas no lado $1, E_{2}$ é a energia de interação entre as partículas no lado 2 e $E_{12}$ é a energia de interação entre as partículas de lados diferentes, partículas próximas à superfície que separa os subsistemas.

Em um sistema com interação de curto alcance (figura (3.2)), as partículas interagem com suas vizinhas diretas apenas. Calculando a energia de interação entre as partículas do lado 1 (linha azul contínua) $E_{1}$, a energia de interação entre as partículas no lado 2 (linha azul contínua) $E_{2}$ e a energia de interação entre as partículas de subsistemas diferentes, ou seja, as partículas que se encontram próximas à superfície que separa os subsistemas (linha pontilhada vermelha) $E_{12}$, vamos considerar o limite $N \rightarrow \infty$. Nesse limite a interação entre as partículas próximas ao vínculo é muito pequena, em comparação com o número de interações entre as partículas nos subsistemas 1 e 2. Então a energia total do sistema será dada por:

$$
E_{1}+E_{2} \approx E
$$

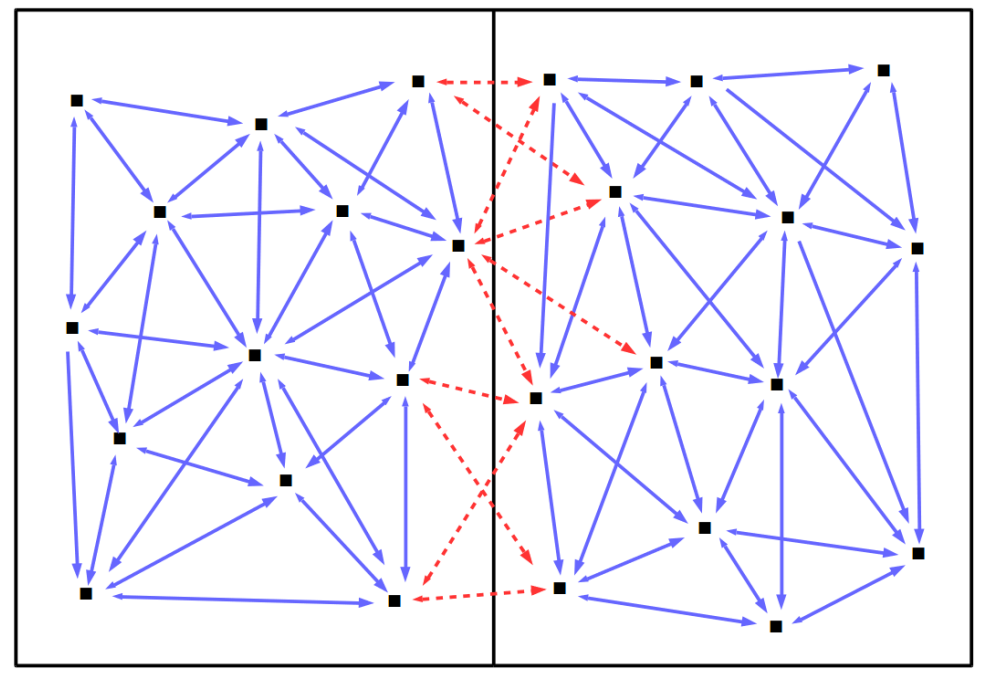

Figura 3.2: Modelo de sistema com interação de curto alcance entre as partículas (Imagem retirada da dissertação de mestrado [8]).

Porém, para um sistema com interação de longo alcance, figura (3.3), todas as partículas interagem entre si, não importa o lado em que estão, logo a energia de interação entre as partículas de lados diferentes (linha vermelha pontilhada) não pode mais ser 
ignorada e a energia total do sistema é dada por:

$$
E_{1}+E_{2}+E_{12}=E
$$

Dessa forma o sistema não é mais aditivo, a soma das energias de interação de cada lado não é igual ao total da energia do sistema.

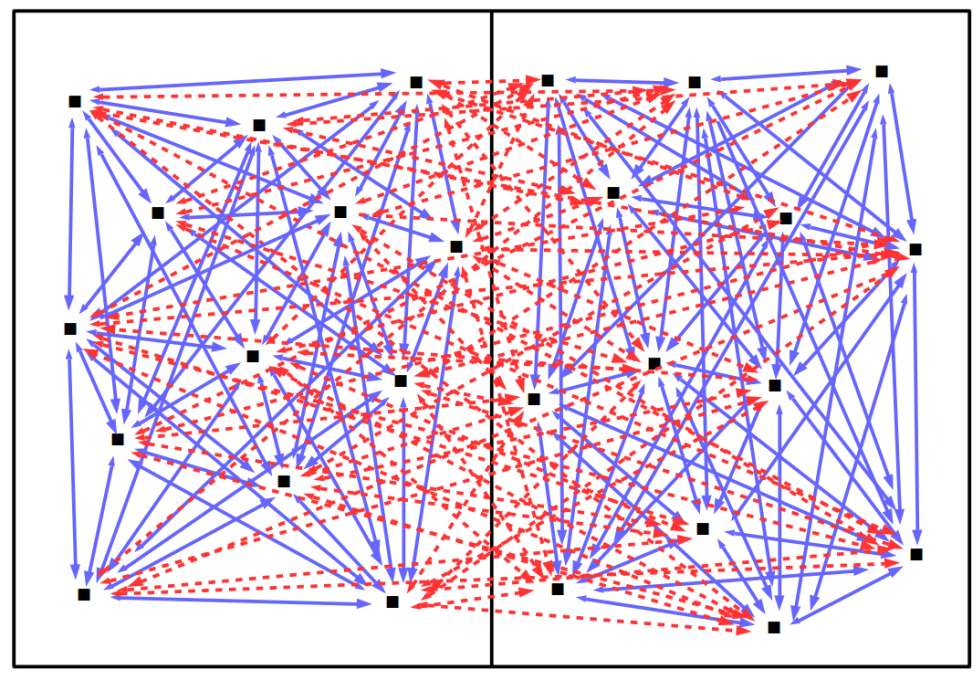

Figura 3.3: Modelo de sistema com interação de longo alcance entre as partículas (Imagem retirada da dissertação de mestrado [8]).

\subsubsection{A inequivalência entre os ensembles}

A não aditividade tem algumas consequências na construção de outros ensembles termodinâmicos, a partir de um ensemble em estudo.

Em sistemas com interações de curto alcance, os ensembles estatísticos são equivalentes. A equivalência entre os ensembles possui duas propriedades importantes [8]:

- No limite termodinâmico, as flutuações dos parâmetros termodinâmicos que não estão fixados desaparecem;

- Um macroestado que pode ser realizado em um ensemble também pode ser realizado em outro ensemble.

Usualmente, dado um sistema físico descrito no ensemble microcanônico e composto de dois subsistemas $(1,2)$, com o sistema 1 bem menor que o sistema 2 (reservatório), podemos escrever o número de estados acessíveis a esse sistema da seguinte forma: 


$$
P(E)=\int d E_{2} \Omega\left(E_{2}\right) \delta\left(E_{1}+E_{2}+E_{12}-E\right) .
$$

Se as interações são de curto alcance $E_{12}=0$ então:

$$
P(E) \propto \Omega\left(E_{2}\right)=\Omega\left(E-E_{1}\right) .
$$

Utilizando a relação $S_{2}=k_{B} \ln \Omega_{2}$ obtemos:

$$
P(E) \propto e^{\frac{S_{2}\left(E-E_{1}\right)}{k_{B}}}
$$

expandindo em série de Taylor o expoente $\frac{S_{2}\left(E-E_{1}\right)}{k_{B}}$ :

$$
P(E) \propto \Omega_{2}\left(E_{2}\right) e^{-\beta E} .
$$

Essa é a distribuição usual no ensemble canônico. Sendo $\Omega_{1}$ os estados acessíveis no sistema 1 e $\Omega_{2}$ os estados acessíveis no sistema 2 .

Essa é uma ligação entre os ensembles canônico e microcanônico e mostra que existe equivalência entre os dois, porém ela depende diretamente da aditividade da energia, $E_{1}+E_{2}=E$.

Em sistemas não aditivos, portanto, nem sempre temos a equivalência entre os ensembles, e a curva do gráfico da entropia pela energia nesses sistemas pode ter uma convexidade o que aponta a existência de calor específico $\left(c_{v}\right)$ negativo.

Para exemplificar melhor o conceito de calor específico negativo, considere dois subsistemas idênticos e aditivos, cada um com energia $E$ e entropia $S(E)$, separados por uma parede que impede a transferência de energia [13]. Dessa forma, sabemos que a energia total é dada por (sendo o sistema aditivo):

$$
E_{T}=E_{1}+E_{2}=2 E
$$

e a entropia toral:

$$
S_{T}=S_{1}\left(E_{1}\right)+S_{2}\left(E_{2}\right)=2 S
$$

$\operatorname{com} E_{1}=E_{2}$ e $S_{1}=S_{2}$.

Removendo a parede e um dos sistemas cedendo energia $(\delta E)$ para o outro, então a nova entropia será: 


$$
S_{T}=S(E+\delta E)+S(E-\delta E)
$$

e a segunda Lei da Termodinâmica nos impõe que:

$$
S(E+\delta E)+S(E-\delta E) \leq 2 S
$$

No limite $\delta E \rightarrow 0$ a equação anterior pode ser escrita como:

$$
\left(\frac{\partial^{2} S}{\partial E^{2}}\right)_{V, N} \leq 0
$$

e sendo $\left(\frac{\partial^{2} S}{\partial E^{2}}\right)_{V, N}=-\frac{1}{\left(T^{2} c_{v}\right)}$, garantimos que

$$
c_{v} \geq 0
$$

Podemos concluir, então, que para sistemas aditivos é impossível a existência de calor específico negativo. Porém, para sistemas não aditivos, há essa possibilidade e quando existe calor específico negativo os ensembles são inequivalentes.

Outro exemplo de fácil entendimento do conceito de calor específico negativo é o sistema auto-gravitante. O teorema do virial para essas partículas [5] é:

$$
2<E_{c}>+<E_{p o t}>=0
$$

com $<E_{c}>$ e $<E_{\text {pot }}>$ representando as energias cinética e potencial, respectivamente.

A energia total é dada por:

$$
E=<E_{c}>+<E_{p o t}>=-<E_{c}>
$$

e sendo a energia cinética média proporcional à temperatura

$$
c_{v}=\frac{\partial E}{\partial T} \propto \frac{\partial E}{\partial E_{c}}<0
$$

ao dar energia para o sistema auto gravitante, os corpos se distanciam e o equivalente a energia cinética diminui. 


\subsection{Teoria de Campo Médio}

Em um sistema com interação de longo alcance as partículas estudadas não interagem apenas com seus vizinhos diretos, como normalmente estudamos, mas interagem todas

entre si. Nessa configuração é possível existir uma ordenação de longo alcance, ou seja, ao perturbar uma partícula estaremos perturbando todas as outras do sistema.

A teoria de Campo Médio é uma aproximação importante no estudo de sistemas com interação de longo alcance, porém não inclui flutuações. O que a torna boa descritora qualitativa do comportamento da transição de fase contínua, mas é inadequada para descrever o sistema na região crítica. Se alguma parte do sistema se afasta do comportamento médio, o que acontece na região crítica, a teoria de campo médio não poderá descrevêla. Quanto maior a dimensão do sistema, mais difícil é para as flutuações arruinarem as correlações, portanto, em dimensões maiores que 4 a teoria de campo médio é muito bem aplicada para estudos de transição de fase contínua [14].

Essa teoria considera que a interação entre cada partícula tem a mesma intensidade. O que fazemos é concentrar nossa atenção em uma das partículas e observar o conjunto de todos os seus vizinhos através de um valor médio da força de interação. Assim, podemos estudar a interação entre a partícula e a média desse conjunto.

A Teoria de Landau é um exemplo de teoria de campo médio pois a energia livre é expandida em termos da magnetização, ou seja, a média simples dos spins, ao invés de expandir em termos da média ponderada dos spins considerando o fator estatístico de Boltzmann.

Os modelos com a mesma expansão de Landau compartilham os mesmos expoentes críticos. Então, teoria de campo médio leva a uma classe de universalidade.

Nesse capítulo estudamos um sistema de campo médio e nos próximos capítulos calculamos seu expoente crítico $\alpha$ com o intuito de comparar o valor obtido com o previsto pela teoria de Landau.

\subsubsection{Hamiltoniano de Campo Médio (Hamiltonian Mean Field - HMF)}

O modelo descrito pelo Hamiltoniano de Campo Médio é interpretado como um sistema de partículas dispostas em um círculo de raio unitário [8], uma forma de representação é a mostrada na figura (3.4). Cada partícula interage com todas as outras e se 
dispõe em um campo de força que, a cada tempo, é a soma dos campos de força individuais de cada outra partícula [15]. Pode ser interpretado também como um sistema de spins em uma fileira, onde todos os vizinhos interagem entre si e assumem valores +1 ou $-1[15]$.

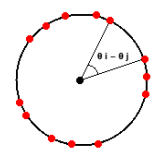

Figura 3.4: Possível representação do HMF.

O Hamiltoniano de Campo Médio é dado por:

$$
H_{N}=\sum_{i=1}^{N} \frac{\left(p_{i}\right)^{2}}{2}+\frac{\varepsilon \sum_{i, j=1}^{N}\left[1-\cos \left(\theta_{i}-\theta_{j}\right)\right]}{2 N}=K+U
$$

sendo que $\theta_{i, j} \in[0,2 \pi)$ refere-se às posições das partículas $i$ e $j, p_{i}$ é o momento da partícula $i$ e $\varepsilon$ é uma constante de acoplamento.

Essa hamiltoniana descreve a soma da energia cinética $(K)$ e potencial $(U)$ do sistema, na energia potencial notamos o caráter de longo alcance do sistema, todas as partículas interagem entre si.

A magnetização de um sistema descrito pelo Hamiltoniano de Campo Médio é escrita como:

$$
m_{y}=\frac{1}{N} \sum_{i=1}^{N} \operatorname{sen} \theta_{i}, m_{x}=\frac{1}{N} \sum_{i=1}^{N} \cos \theta_{i}
$$

$\mathrm{e}$

$$
m^{2}=m_{x}^{2}+m_{y}^{2}
$$

ou seja, a magnetização do sistema está associada à posição das partículas no anel.

Podemos reescrever a equação (3.9) em termos da magnetização: 


$$
\begin{aligned}
H_{N} & =\sum_{i=1}^{N} \frac{\left(p_{i}\right)^{2}}{2}+\frac{\varepsilon \sum_{i, j=1}^{N}\left[1-\cos \left(\theta_{i}-\theta_{j}\right)\right]}{2 N}= \\
& =\sum_{i=1}^{N} \frac{p_{i}^{2}}{2}+\frac{\varepsilon}{2 N} N^{2}-\frac{\varepsilon}{2 N} \sum_{i, j=1}^{N}\left[\cos \left(\theta_{i}-\theta_{j}\right)\right]= \\
& =\sum_{i=1}^{N} \frac{p_{i}^{2}}{2}+\frac{\varepsilon N}{2}-\frac{\varepsilon}{2 N} \sum_{i, j=1}^{N}\left[\cos \left(\theta_{i}\right) \cos \left(\theta_{j}\right)+\operatorname{sen}\left(\theta_{i}\right) \operatorname{sen}\left(\theta_{j}\right)\right]= \\
& =\sum_{i=1}^{N} \frac{p_{i}^{2}}{2}+\frac{\varepsilon N}{2}-\frac{\varepsilon}{2 N} \sum_{i=1}^{N}\left[\cos ^{2}\left(\theta_{i}\right)+\operatorname{sen}^{2}\left(\theta_{i}\right)\right]= \\
& =\sum_{i=1}^{N} \frac{p_{i}^{2}}{2}+\frac{\varepsilon N}{2}-\frac{\varepsilon}{2 N} N^{2} m^{2}= \\
& =\sum_{i=1}^{N} \frac{p_{i}^{2}}{2}+\frac{\varepsilon N}{2}\left(1-m^{2}\right)
\end{aligned}
$$

A partir das equações de Hamilton:

$$
\dot{\theta}=\frac{\partial H_{N}}{\partial p}
$$

$\mathrm{e}$

$$
\dot{p}=-\frac{\partial H_{N}}{\partial \theta},
$$

podemos calcular a força que age sobre uma partícula $i$, usando a equação (3.12):

$$
\begin{aligned}
F_{i} & =-\frac{\partial\left(\sum_{i=1}^{N} \frac{\left(p_{i}\right)^{2}}{2}+\frac{\varepsilon \sum_{i, j=1}^{N}\left[1-\cos \left(\theta_{i}-\theta_{j}\right)\right]}{2 N}\right)}{\partial \theta_{r}}= \\
& =-\frac{\varepsilon}{N} \sum_{j} \operatorname{sen}\left(\theta_{i}-\theta_{j}\right)= \\
& =-\varepsilon M \operatorname{sen}\left(\theta_{i}-\phi\right),
\end{aligned}
$$

sendo $\vec{m}=\frac{1}{N} \vec{M}=\left(m_{x}, m_{y}\right)$ e $\phi=\tan ^{-1}\left(\frac{m_{y}}{m_{x}}\right)$ é a fase.

Isso mostra que, a cada tempo, a força que age no sistema é a soma dos campos de força individuais das outras partículas. O movimento de cada partícula é determinado pela evolução temporal de M, que depende do movimento de todas as partículas [15]. E o sistema pode ser interpretado, também, como um conjunto de pêndulos acoplados. 


\section{Capítulo 4}

\section{Estudo do Hamiltoniano de Campo}

\section{Médio - HMF}

Relembrando que o Hamiltoniano de Campo Médio é dado por:

$$
H_{N}=\sum_{i=1}^{N} \frac{\left(p_{i}\right)^{2}}{2}+\frac{\varepsilon \sum_{i, j=1}^{N}\left[1-\cos \left(\theta_{i}-\theta_{j}\right)\right]}{2 N}
$$

sendo que $\theta_{i, j} \in[0,2 \pi)$ refere-se às posições das partículas $i$ e $j, p_{i}$ é o momento da partícula $i$ e $\varepsilon$ é uma constante de acoplamento.

Vamos estudar o sistema nos ensembles microcanônico e canônico a partir das funções termodinâmicas encontradas para cada um deles.

\subsection{Ensemble microcanônico}

No ensemble microcanônico o número de configurações microscópicas (microestados) possíveis, para um sistema com uma dada uma energia $E$ e número $N$ de partículas, é dado por:

$$
W_{N}(E)=\int \delta\left(E-H_{N}\right) \prod_{i=1}^{N} d p_{i} d \theta_{i}
$$

com $H_{N}$ dado pela equação (4.1). Podemos reescrever a equação acima na forma:

$$
W_{N}(E)=\int \prod_{i=1}^{N} d p_{i} d \theta_{i} \int d K \delta\left(K-\sum_{j=1}^{N} \frac{p_{j}^{2}}{2}\right) \delta\left(E-K-U\left(\left\{\theta_{i}\right\}\right)\right)
$$

dado que $\int d K \delta\left(K-\sum_{j=1}^{N} \frac{p_{j}^{2}}{2}\right)=1$, obtemos: 


$$
W_{N}(E)=\int d K \int \prod_{i=1}^{N} d p_{i} \delta\left(K-\sum_{j=1}^{N} \frac{p_{j}^{2}}{2}\right) \int \prod_{i=1}^{N} d \theta_{i} \delta\left(E-K-U\left(\left\{\theta_{i}\right\}\right)\right)
$$

$\mathrm{Ou}$

$$
W_{N}(E)=\int d K W_{\text {cin }}(K) W_{\text {conf }}(E-K)
$$

sendo

$$
W_{\text {cin }}(K)=\int \prod_{i=1}^{N} d p_{i} \delta\left(K-\sum_{j=1}^{N} \frac{p_{j}^{2}}{2}\right)
$$

o número de estados acessíveis dado $K$, energia cinética, e

$$
W_{\text {conf }}(E-K)=\int \prod_{i=1}^{N} d \theta_{i} \delta\left(E-K-U\left(\left\{\theta_{i}\right\}\right)\right),
$$

o número de estados acessíveis dado $E-K$.

Para continuar o cálculo vamos usar o método da transformada de Laplace. Esse método permite levar a resolução de equações diferenciais à resolução de equações algébricas, que são muito mais simples de resolver. A transformada de Laplace de uma função $f(x)$, cuja integral existe para $x \geq 0$ e real, é a função $\mathrm{F}(\mathrm{s})$, definida por:

$$
F(s)=\mathcal{L}\{f\}(s)=\int_{0}^{\infty} e^{-s x} f(x) d x
$$

Calculando a transformada de Laplace de $W_{\text {cin }}(K)$ com respeito a $\beta=\frac{1}{k_{B} T}$, (com $F(s)=Z_{\text {cin }}(\beta), f(x)=W_{\text {cin }}(K), s=\beta$ e $\left.x=K\right)$, temos: 


$$
\begin{aligned}
Z_{\text {cin }}(\beta) & =\int_{0}^{\infty} e^{-\beta K} W_{\text {cin }}(K) d K= \\
& =\int_{0}^{\infty} \int_{-\infty}^{\infty} d K \prod_{i=1}^{N} d p_{i} e^{-\beta K} \delta\left(K-\sum_{j=1}^{N} \frac{p_{j}^{2}}{2}\right)= \\
& =\int_{-\infty}^{\infty} \prod_{i=1}^{N} d p_{i} \exp \left(-\beta \sum_{j=1}^{N} \frac{p_{j}^{2}}{2}\right)= \\
& =\int_{-\infty}^{\infty} \prod_{i=1}^{N} d p_{i} \prod_{j=1}^{N} \exp \left(-\beta \frac{p_{j}^{2}}{2}\right)= \\
& =\int_{-\infty}^{\infty} d p_{1} \exp \left(-\beta \frac{p_{1}^{2}}{2}\right) \int_{-\infty}^{\infty} d p_{2} \exp \left(-\beta \frac{p_{2}^{2}}{2}\right) \cdots \int_{-\infty}^{\infty} d p_{N} \exp \left(-\beta \frac{p_{N}^{2}}{2}\right)= \\
& =\left[\int_{-\infty}^{\infty} d p \exp \left(-\beta \frac{p^{2}}{2}\right)\right]^{N},
\end{aligned}
$$

sendo $Z_{\text {cin }}(\beta)$ a função de partição canônica cinética associada, via transformada de Laplace, ao número de estados acessíveis $W_{\text {cin }}(K)$.

Então,

$$
Z_{\text {cin }}(\beta)=\left(\frac{2 \pi}{\beta}\right)^{\frac{N}{2}}
$$

Aplicando a transformada inversa de Laplace $\mathcal{L}^{-1}\left\{\frac{\Gamma(n+1)}{s^{n+1}}\right\}=t^{n} \Theta(t)$, em (4.8), obtemos

$$
\begin{aligned}
W_{\text {cin }}(K) & =\mathcal{L}^{-1}\left\{Z_{\text {cin }}(\beta)\right\}=\mathcal{L}^{-1}\left\{\left(\frac{2 \pi}{\beta}\right)^{\frac{N}{2}}\right\}= \\
& =\frac{(2 \pi)^{\frac{N}{2}}}{\Gamma\left(\frac{N}{2}\right)} \mathcal{L}^{-1}\left\{\frac{\Gamma\left(\frac{N}{2}\right)}{\beta^{\frac{N}{2}}}\right\}=\frac{(2 \pi)^{\frac{N}{2}}}{\Gamma\left(\frac{N}{2}\right)} K^{\frac{N}{2}-1} \Theta(K)
\end{aligned}
$$

como $K \geq 0$,

$$
W_{\text {cin }}(K)=\frac{(2 \pi)^{\frac{N}{2}}}{\Gamma\left(\frac{N}{2}\right)} K^{\frac{N}{2}-1}
$$

e

$$
\ln W_{\text {cin }}(K)=\frac{N}{2} \ln 2 \pi+\left(\frac{N}{2}-1\right) \ln K-\ln \Gamma\left(\frac{N}{2}\right) .
$$

Usando a expressão assintótica da função $\Gamma, \ln \Gamma(N) \simeq\left(N-\frac{1}{2}\right) \ln N-N+\frac{1}{2} \ln 2 \pi$, em (4.9), 


$$
\ln W_{\text {cin }}(K) \simeq \frac{N}{2}\left[\ln 2 \pi+\left(1-\frac{2}{N}\right) \ln K-\left(1-\frac{1}{N}\right) \ln \frac{N}{2}+1-\frac{1}{N} \ln 2 \pi\right]
$$

Desprezando os termos que vão a zero no limite em que $N \rightarrow \infty$, a equação (4.10) se torna

$$
\ln W_{c i n}(K) \simeq \frac{N}{2}\left[1+\ln 4 \pi+\ln \frac{K}{N}\right]
$$

$\mathrm{e}$

$$
W_{\text {cin }}(K) \simeq \exp \left[\frac{N}{2}\left(1+\ln 4 \pi+\ln \frac{K}{N}\right)\right] .
$$

Substituindo (4.11) em (4.5), obtemos

$$
W_{N}(E)=\int d K \exp \left[\frac{N}{2}\left(1+\ln 4 \pi+\ln \frac{K}{N}\right)\right] W_{c o n f}(E-K) .
$$

Definindo a densidade de energia total como $e=\frac{E}{N}$, a densidade da energia cinética como $k=\frac{K}{N}$ e a densidade da potencial como $u=\frac{U}{N}$. E definindo também a entropia configuracional como $s_{\text {conf }}(e-k)=\frac{1}{N} \ln W_{\text {conf }}(E-K)$, onde $e-k=u$. A função de partição microcanônica (4.12) pode ser reescrita como:

$$
W_{N}(N e)=N \int d k \exp \left[N\left(\frac{1}{2}+\frac{1}{2} \ln 4 \pi+\frac{1}{2} \ln k+s_{\text {conf }}(e-k)\right)\right]
$$

A expressão (4.13) fornece o número de microestados do sistema com uma energia total $E=N e$. A integração em $k$ equivale à soma de todos os microestados com energia total $E=N e$ e energia cinética $K=N k$, ou seja,

$$
W_{N}(N e)=\int d(N k) W_{N}(N e, N k)
$$

Comparando (4.14) com (4.13), temos que

$$
W_{N}(N e, N k)=\exp \left[N\left(\frac{1}{2}+\frac{1}{2} \ln 4 \pi+\frac{1}{2} \ln k+s_{\text {conf }}(e-k)\right)\right] .
$$

Substituindo $k=e-u$, em (4.15), temos o número de microestados do sistema com energia total $E=N e$ e energia potencial $U=N u$, 


$$
W_{N}(N e, N u)=\exp \left[N\left(\frac{1}{2}+\frac{1}{2} \ln 4 \pi+\frac{1}{2} \ln (e-u)+s_{\text {conf }}(u)\right)\right] .
$$

A entropia por partícula, em função de $e$ e $u$, é obtida aplicando o limite termodinâmico

$$
s(e, u)=\lim _{N \rightarrow \infty} \frac{1}{N} \ln W_{N}(N e, N u)=\frac{1}{2}+\frac{1}{2} \ln 4 \pi+\frac{1}{2} \ln (e-u)+s_{\text {conf }}(u) .
$$

Uma forma de se obter $s(e)$ é maximizando a expressão (4.17) em relação a $u$, para isso é necessário ter uma expressão explícita da entropia configuracional $s_{\text {conf }}(u)$, ou seja, é preciso calcular $W_{\text {conf }}(U)$.

No caso do HMF-1D, da equação (3.10), $U$ e $|m|$ possuem uma correspondência um para um, ou seja, definindo

$$
W(m)=\int \prod_{i} d \theta_{i} \delta\left(\sum_{i} \cos \theta_{i}-N m\right) \delta\left(\sum_{i} \sin \theta_{i}\right),
$$

temos que $W(m)$ é proporcional a $W_{\text {conf }}(U)$ (dada a correspondência um para um). Vamos definir a magnetização na direção do eixo $x$, como foi feito na expressão (4.18). Usando a representação de Fourier da função $\delta$ em (4.18), temos

$$
\begin{aligned}
W(m)= & \left(\frac{1}{2 \pi}\right)^{2} \int_{-\infty}^{\infty} d q_{2} \int_{-\infty}^{\infty} d q_{1} \int \prod_{i} d \theta_{i} \exp \left[i q_{1}\left(\sum_{i} \cos \theta_{i}-N m\right)\right] \times \\
& \times \exp \left[i q_{2}\left(\sum_{i} \sin \theta_{i}\right)\right]= \\
= & \left(\frac{1}{2 \pi}\right)^{2} \int_{-\infty}^{\infty} d q_{2} \int_{-\infty}^{\infty} d q_{1} \int \prod_{i} d \theta_{i} e^{-i q_{1} N m} \exp \left(i q_{1} \sum_{i} \cos \theta_{i}+i q_{2} \sum_{i} \sin \theta_{i}\right)= \\
= & \left(\frac{1}{2 \pi}\right)^{2} \int_{-\infty}^{\infty} d q_{2} \int_{-\infty}^{\infty} d q_{1} \int \prod_{i} d \theta_{i} e^{-i q_{1} N m} \exp \left[\sum_{i}\left(i q_{1} \cos \theta_{i}+i q_{2} \sin \theta_{i}\right)\right]= \\
= & \left(\frac{1}{2 \pi}\right)^{2} \int_{-\infty}^{\infty} d q_{2} \int_{-\infty}^{\infty} d q_{1} e^{-i q_{1} N m} \int_{i} d \theta_{i}\left[e^{i q_{1} \cos \theta_{1}+i q_{2} \sin \theta_{1}}\right] \cdots\left[e^{\left.i q_{1} \cos \theta_{N}+i q_{2} \sin \theta_{N}\right]}\right. \\
= & \left(\frac{1}{2 \pi}\right)^{2} \int_{-\infty}^{\infty} d q_{1} e^{-i q_{1} N m} \int_{-\infty}^{\infty} d q_{2}\left[\int_{0}^{2 \pi} d \theta e^{i\left(q_{1} \cos \theta+q_{2} \sin \theta\right)}\right]^{N} \cdot
\end{aligned}
$$

Usando $a \cos x+b \sin x=R \cos (x-\alpha)$, onde $R=\sqrt{a^{2}+b^{2}}$ e $\alpha=\tan ^{-1}\left(\frac{b}{a}\right)$, obtemos 


$$
W(m)=\left(\frac{1}{2 \pi}\right)^{2} \int_{-\infty}^{\infty} d q_{1} \int_{-\infty}^{\infty} d q_{2} e^{-i q_{1} N m} J_{0}^{N}\left(\left(q_{1}^{2}+q_{2}^{2}\right)^{\frac{1}{2}}\right) .
$$

$J_{0}(z)$ é a função de Bessel de ordem zero:

$$
J_{0}(z)=\int_{0}^{2 \pi} d \theta e^{i z \cos \theta}
$$

Com isso, podemos escrever (4.20) na forma

$$
W(m)=\left(\frac{1}{2 \pi}\right)^{2} \int_{-\infty}^{\infty} \int_{-\infty}^{\infty} d q_{1} d q_{2} e^{N f\left(q_{1}, q_{2}\right)},
$$

$\operatorname{com} f\left(q_{1}, q_{2}\right)=-i q_{1} m+\ln J_{0}\left(\left(q_{1}^{2}+q_{2}^{2}\right)^{\frac{1}{2}}\right)$. Podemos aproximar a integral em (4.22) pelo valor máximo do integrando, de acordo com o saddle point method ${ }^{1}$. Usando o fato de que a derivada de $J_{0}$ é $-J_{1}$ (função de Bessel de ordem um), para achar $q_{1}^{*}$ e $q_{2}^{*}$, que maximizam $f\left(q_{1}, q_{2}\right)$, temos que resolver o seguinte sistema:

$$
\frac{\partial f}{\partial q_{1}}=0
$$

$\mathrm{e}$

$$
\frac{\partial f}{\partial q_{2}}=0
$$

Temos como resolução as seguintes equações:

$$
\begin{gathered}
i m+\frac{J_{1}\left(\left(q_{1}^{* 2}+q_{2}^{* 2}\right)^{\frac{1}{2}}\right)}{J_{0}\left(\left(q_{1}^{* 2}+q_{2}^{* 2}\right)^{\frac{1}{2}}\right)} \frac{q_{1}^{*}}{\sqrt{q_{1}^{* 2}+q_{2}^{* 2}}}=0, \\
\frac{J_{1}\left(\left(q_{1}^{* 2}+q_{2}^{* 2}\right)^{\frac{1}{2}}\right)}{J_{0}\left(\left(q_{1}^{* 2}+q_{2}^{* 2}\right)^{\frac{1}{2}}\right)} \frac{q_{2}^{*}}{\sqrt{q_{1}^{* 2}+q_{2}^{* 2}}}=0 .
\end{gathered}
$$

Da equação (4.24), temos que $q_{2}^{*}=0$, com isso a equação (4.23) fica

$$
\frac{J_{1}\left(q_{1}^{*}\right)}{J_{0}\left(q_{1}^{*}\right)}=-i m
$$

Definindo $q_{1}^{*}=-i \gamma$ e usando a propriedade das funções de Bessel $I_{n}(z)=i^{-n} J_{n}(i z)$, onde $I_{n}(z)$ é a função modificada de Bessel de ordem $n$, obtemos

$$
\frac{I_{1}(\gamma)}{I_{0}(\gamma)}=m
$$

\footnotetext{
${ }^{1}$ Dado a integral $I_{N}=\int d x e^{N f(x)} ;$ se $f$ é contínua, então $\lim _{N \rightarrow \infty} I_{N}=\max _{x} e^{f(x)}[16]$.
} 
Denotando por $B_{i n v}$ a função inversa de $\frac{I_{1}}{I_{0}}$, temos que $q_{1}^{*}=-i B_{i n v}(m)$. Com isso,

$$
W(m) \simeq\left(\frac{1}{2 \pi}\right)^{2} e^{N\left[-m B_{i n v}(m)+\ln I_{0}\left(B_{i n v}(m)\right)\right]},
$$

$\mathrm{e}$

$$
s_{\text {conf }}(m)=\lim _{N \rightarrow \infty} \frac{1}{N} \ln W(m)=-m B_{\text {inv }}(m)+\ln I_{0}\left(B_{\text {inv }}(m)\right) .
$$

Substituindo (4.28) em (4.17) e usando $u=\frac{1}{2}-\frac{1}{2} m^{2}$, temos que a entropia por partícula em função de $e$ e $m$ é dada por

$$
s(e, m)=\frac{1}{2}(1+\ln 4 \pi)+\frac{1}{2} \ln \left(e-\frac{1}{2}+\frac{1}{2} m^{2}\right)-m B_{i n v}(m)+\ln I_{0}\left(B_{i n v}(m)\right) .
$$

De (4.29), temos

$$
\begin{gathered}
\frac{\partial s(e, m)}{\partial m}=\frac{m}{2 e-1+m^{2}}-B_{i n v}(m) \\
\frac{\partial s(e, m)}{\partial e}=\frac{1}{2 e-1+m^{2}}
\end{gathered}
$$

A magnetização espontânea $m(e)$ é obtida igualando a equação (4.30) a zero, ou seja, ela deve satisfazer

$$
\frac{m(e)}{2 e-1+m^{2}(e)}-B_{i n v}(m(e))=0 .
$$

Uma vez obtida a entropia $s(e)$, a temperatura do sistema é dada por (utilizando as equações $(4.30),(4.31)$ e (4.32))

$$
\begin{aligned}
\frac{d s(e)}{d e}=\frac{1}{T(e)} & =\left[\frac{\partial s(e, m)}{\partial e}+\frac{\partial s(e, m)}{\partial m} \frac{\partial m}{\partial e}\right]_{m=m(e)}= \\
& =\left[\frac{\partial s(e, m)}{\partial e}+\left(\frac{m}{2 e-1+m^{2}}-B_{i n v}(m)\right) \frac{\partial m}{\partial e}\right]_{m=m(e)}= \\
& =\left[\frac{\partial s(e, m)}{\partial e}\right]_{m=m(e)}
\end{aligned}
$$

$$
T(e)=2 e-1+m^{2}(e)
$$




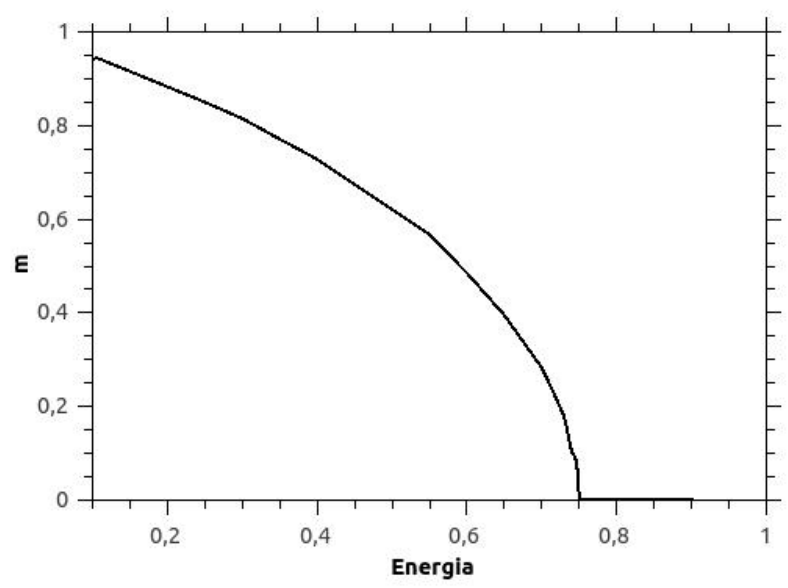

Figura 4.1: O gráfico da magnetização pela entropia, 4.1, nos mostra a existência de uma transição de fase contínua nesse sistema em $e=0,75$.

A curva calórica do sistema é dada pela figura (4.2).

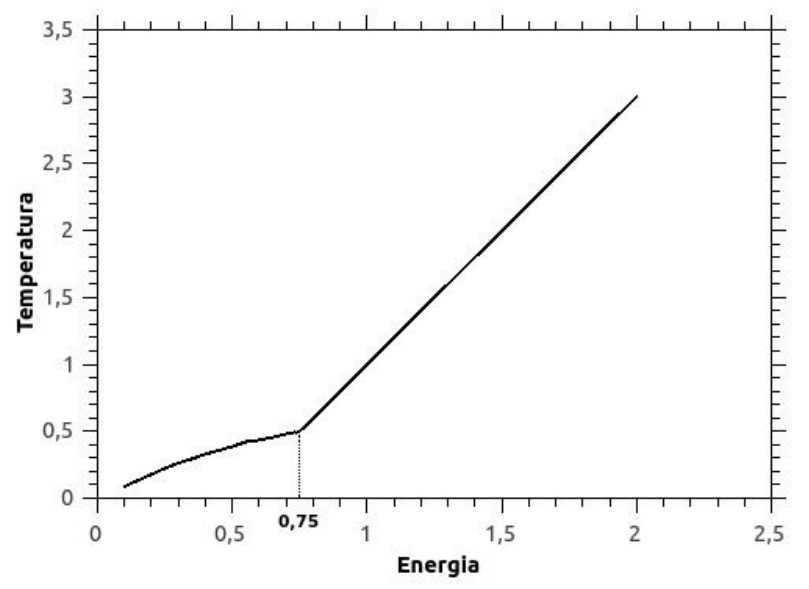

Figura 4.2: Curva calórica para o HMF microcanônico

Podemos plotar o gráfico do calor específico pela energia derivando a curva calórica, figura (4.2), e observamos uma descontinuidade finita no calor específico, figura (4.3), que nos indica, também, uma transição de fase contínua. 


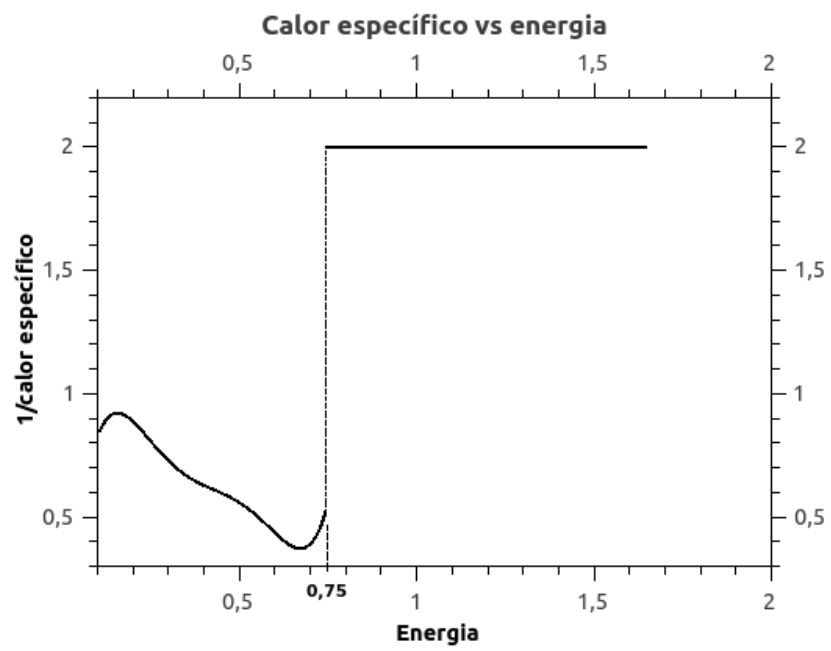

Figura 4.3: Calor específico versus energia para o HMF

\subsection{Ensemble canônico}

A função de partição canônica é dada por:

$$
\begin{aligned}
Z(\beta, N) & =\int \mathrm{e}^{-\beta H_{N}(\theta, p)} d \theta \mathrm{d} p= \\
& =\int \prod_{i=1}^{N} \mathrm{e}^{-\beta H_{N}\left(\theta_{i}, p_{i}\right)} d \theta_{i} d p_{i} .
\end{aligned}
$$

Com $H_{N}$ dado pela equação (4.1). Introduzindo a equação (4.1) na equação (4.35):

$$
Z=\int \prod_{i=1}^{N} d \theta_{i} d p_{i} e^{-\beta \sum_{i=1}^{N} \frac{p_{i}^{2}}{2}} e^{-\beta \frac{\varepsilon}{2 N} \sum_{i, j=1}^{N}\left[1-\cos \left(\theta_{i}-\theta_{j}\right)\right]},
$$

Separando os termos cinético e potencial da equação (4.36):

$$
Z=\int \prod_{l=1}^{N} d p_{l} e^{-\beta \frac{p_{l}^{2}}{2}} \int_{-\pi}^{\pi} \prod_{i=1}^{N} d \theta_{i} e^{-\beta \frac{\varepsilon}{2 N} \sum_{i, j=1}^{N}\left[1-\cos \left(\theta_{i}-\theta_{j}\right)\right]}=Z_{k}+Z_{V}
$$

sendo:

$$
Z_{k}=\int \prod_{l=1}^{N} d p_{l} e^{-\beta \frac{p_{l}^{2}}{2}}
$$

e

$$
Z_{V}=\int_{-\pi}^{\pi} \prod_{i=1}^{N} d \theta_{i} e^{-\beta \frac{\varepsilon}{2 N} \sum_{i, j=1}^{N}\left[1-\cos \left(\theta_{i}-\theta_{j}\right)\right]}=e^{\frac{-\beta \varepsilon N}{2}} J
$$


Com $J$ expresso na forma:

$$
\begin{aligned}
J & =\int_{-\pi}^{\pi} \prod_{i=1}^{N} d \theta_{i} e^{\beta \frac{\varepsilon}{2 N} \sum_{i, j=1}^{N}\left[\cos \left(\theta_{i}-\theta_{j}\right)\right]}= \\
& =\int_{-\pi}^{\pi} \prod_{i=1}^{N} d \theta_{i} e^{\beta \frac{\varepsilon}{2 N}\left[\sum_{i, j=1}^{N}\left(\cos \theta_{i} \cos \theta_{j}+\operatorname{sen} \theta_{i} \operatorname{sen} \theta_{j}\right)\right]}= \\
& =\int_{-\pi}^{\pi} \prod_{i=1}^{N} d \theta_{i} e^{\beta \frac{\varepsilon}{2 N}\left[\left(\sum_{i=1}^{N} \cos \theta_{i}\right)^{2}+\left(\sum_{i=1}^{N} \operatorname{sen} \theta_{i}\right)^{2}\right]}= \\
& =\int_{-\pi}^{\pi} \prod_{i=1}^{N} d \theta_{i} e^{\beta \frac{\varepsilon}{2 N}\left(\sum_{i=1}^{N} M_{i}\right)^{2}} .
\end{aligned}
$$

Utilizando a transformação de Hubbard-Stratonovich, no caso $\mu>0$ :

$$
e^{a x^{\prime 2}}=\sqrt{\frac{a}{\pi}} \int_{-\infty}^{\infty} e^{\left(-a y^{2}+2 x^{\prime} a y\right)} d \vec{y}
$$

com $a=1, x^{\prime 2}=\frac{\mu x^{2}}{2}, y \in \mathbb{R}^{2}$, e definindo:

$$
\begin{gathered}
\mu=\frac{\beta \varepsilon}{N}, \\
x^{2}=\left(\sum_{i=1}^{N} \vec{M}_{i}\right)^{2},
\end{gathered}
$$

podemos reescrever a transformação (4.41) na seguinte forma:

$$
e^{\left(\frac{\mu}{2} x^{2}\right)}=\frac{1}{\pi} \iint_{-\infty}^{\infty} e^{\left[-(\vec{y})^{2}+\sqrt{2 \mu} x \vec{y}\right] d \vec{y}}
$$

Inserindo a equação (4.42) na equação (4.40), obtemos, usando a relação $a \cos x+$ $b \sin x=R \cos (x-\alpha)$, onde $R=\sqrt{a^{2}+b^{2}}$ e $\alpha=\tan ^{-1}\left(\frac{b}{a}\right)$ : 


$$
\begin{aligned}
J & =\frac{1}{\pi} \int_{-\pi}^{\pi} \prod_{i=1}^{N} d \theta_{i} \iint_{-\infty}^{\infty} d \vec{y} e^{\left[-(\vec{y})^{2}+\sqrt{2 \mu} \sum_{i=1}^{N} \vec{M}_{i} \vec{y}\right]}= \\
& =\frac{1}{\pi}\left\{\int_{-\pi}^{\pi} d \theta e^{\sqrt{2 \mu}\left(y_{1} \cos \theta+y_{2} \operatorname{sen} \theta\right)}\right\}^{N} \iint_{-\infty}^{\infty} d \vec{y} e^{-(\vec{y})^{2}}= \\
& =\frac{1}{\pi}\left\{\int_{-\pi}^{\pi} d \theta e^{\sqrt{2 \mu}(y \cos \theta)}\right\}^{N} \iint_{-\infty}^{\infty} d \vec{y} e^{-(\vec{y})^{2}} .
\end{aligned}
$$

Usando a definição da função de Bessel modificada de ordem zero:

$$
I_{0}(x)=\frac{1}{\pi} \int_{0}^{\pi} d \theta e^{(x \cos \theta)}
$$

sendo $x=\sqrt{2 \mu} y$ na equação (4.43).

Substituindo o valor de $y$ por $y \sqrt{\frac{N}{2 \beta \varepsilon}}$ a equação (4.44) fica:

$$
I_{0}(\sqrt{2 \mu} y)=I_{0}\left(\sqrt{\frac{2 \beta \varepsilon}{N} \cdot \frac{N}{2 \beta \varepsilon} y}\right)=I_{0}(y)
$$

Então, dado $I_{0}(y)$ como:

$$
I_{0}(y)=\frac{1}{\pi} \int_{0}^{\pi} d \theta e^{(y \cos \theta)}
$$

temos que:

$$
\int_{-\pi}^{\pi} d \theta e^{(y \cos \theta)}=2 \pi I_{0}(y)
$$

Podemos escrever a equação (4.47) da forma

$$
\left(2 \pi I_{0}(y)\right)^{N}=e^{N \ln \left(2 \pi I_{0}(y)\right)} .
$$

Substituindo a equação (4.48) na equação (4.43) temos:

$$
J=\frac{1}{\pi} \frac{N}{2 \beta \varepsilon} \iint_{-\infty}^{\infty} d \vec{y} e^{\left(\frac{-y^{2} N}{2 \beta \varepsilon}+N \ln \left(2 \pi I_{0}(y)\right)\right.} .
$$

Aplicando (4.49) na função de partição (4.36), obtemos: 


$$
Z=\left(\frac{2 \pi}{\beta}\right)^{\frac{N}{2}} e^{\frac{-\beta \varepsilon N}{2}} \frac{1}{\pi} \frac{N}{2 \beta \varepsilon} \iint_{-\infty}^{\infty} d \vec{y} e^{\left(\frac{-y^{2} N}{2 \beta \varepsilon}+N \ln \left(2 \pi I_{0}(y)\right)\right.}
$$

A energia de Helmholtz por partícula é definida por:

$$
f=-\frac{1}{N \beta} \ln (Z)
$$

Para $N \rightarrow \infty$, podemos aproximar a integral em (4.50) pelo valor máximo do integrando, de acordo com o saddle point method ${ }^{2}$ :

$$
-\beta f=\frac{1}{2} \ln \left(\frac{2 \pi}{\beta}\right)-\frac{\beta \varepsilon}{2}+{ }_{\text {max }}\left\{\frac{-y^{2}}{2 \beta \varepsilon}+\ln \left(2 \pi I_{0}(y)\right)\right\}
$$

sendo $y$ um parâmetro. Minimizando a energia de Helmholtz em y obtemos a equação:

$$
\left(\frac{\partial(-\beta f)}{\partial y}\right)_{T}=0
$$

com resultado:

$$
\frac{y}{\beta \varepsilon}=\frac{I_{1}(y)}{I_{0}(y)}=m^{*}
$$

$I_{i}$ é a função de Bessel modificada de ordem $i \operatorname{com} i=0,1$.

A equação autoconsistente (4.53) é resolvida graficamente na figura (4.4). Para valores de $\beta \varepsilon>2$ o sistema tem solução com $m^{*} \neq 0$, para valores de $\beta \varepsilon<2$ o sistema só tem a solução $m^{*}=0$

Ao plotar o gráfico de $m^{*}$ pela temperatura (figura (4.5)), observamos o comportamento descrito acima no gráfico (4.4) e podemos comparar com o previsto na figura (1.9).

Portanto, $m^{*}$ é o parâmetro de ordem do sistema, que iremos definir por $m^{3}$. O gráfico (4.5) mostra, também, a existência de transição de fase contínua no sistema, pois o parâmetro de ordem diminui continuamente até atingir o valor zero.

A energia do sistema por partícula (e) pode ser calculada pela equação:

$$
e=\frac{\partial(\beta f)}{\partial \beta}
$$

então, utilizando as equações (4.52) e (4.53) e tomando $k_{B}=1$, temos que:

\footnotetext{
${ }^{2}$ Dado a integral $I_{N}=\int d x e^{N f(x)}$; se $f$ é contínua, então $\lim _{N \rightarrow \infty} I_{N}=\max _{x} e^{f(x)}[16]$.

${ }^{3}$ Usualmente $m$ é denominado de "magnetização" por semelhança de resultados com sistemas magnéticos
} 

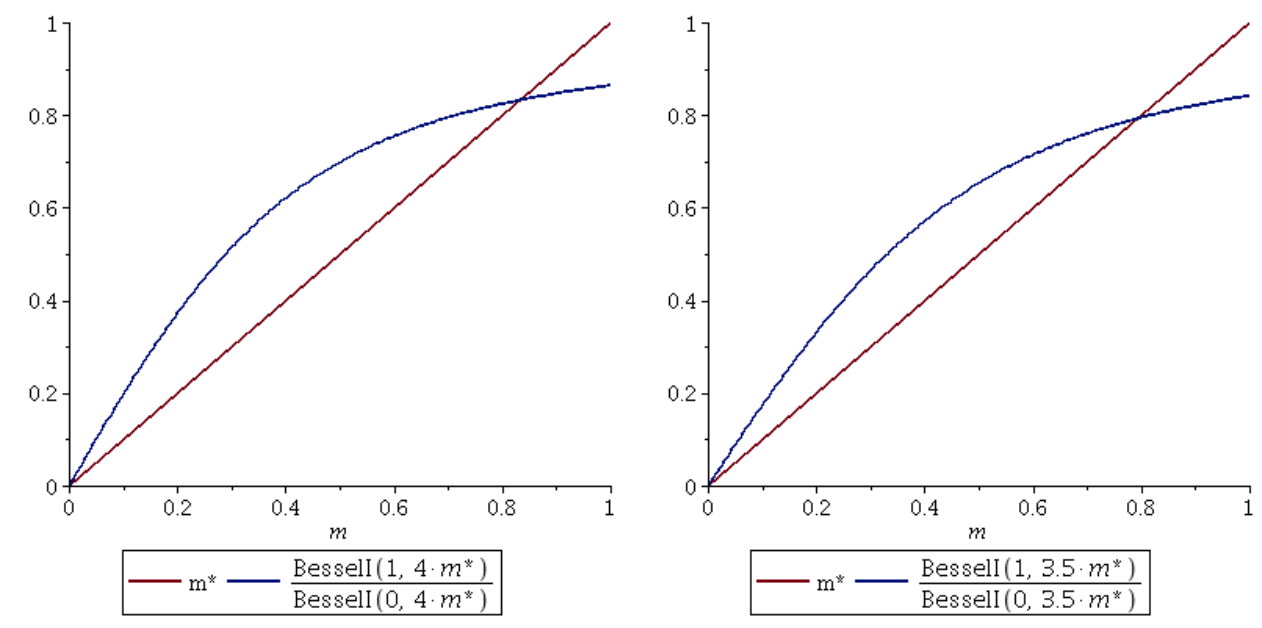

(a) Resolução da equação autoconsistente (b) Resolução da equação autoconsistente para $\beta \varepsilon=4$. para $\beta \varepsilon=3.5$
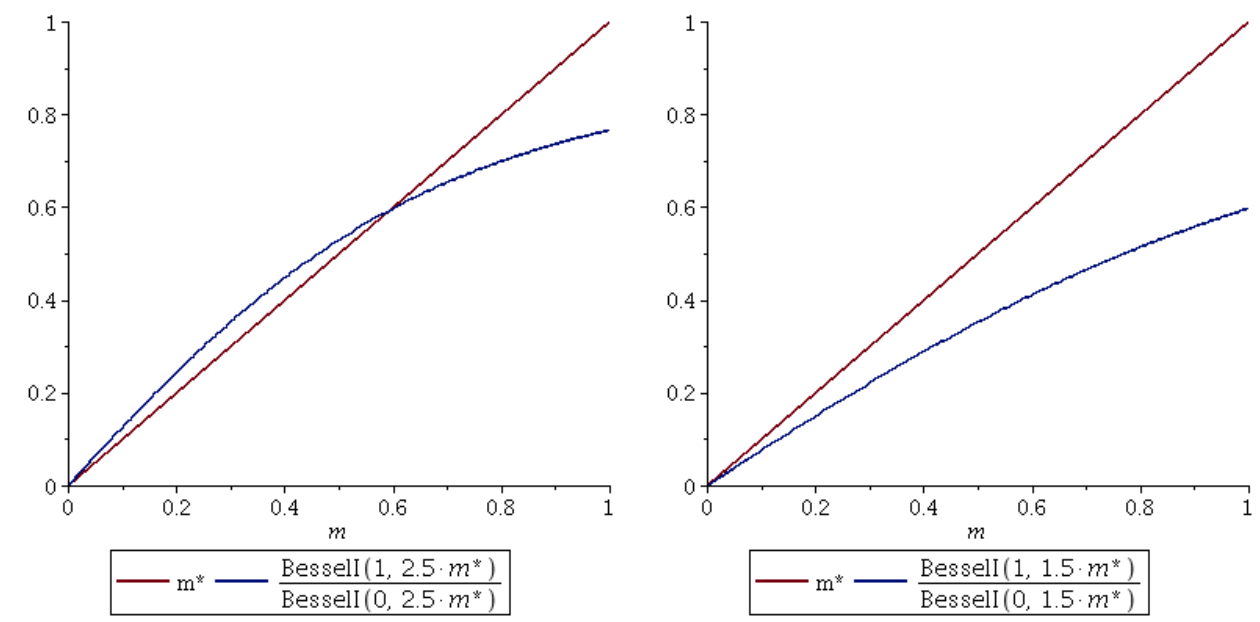

(c) Resolução da equação autoconsistente (d) Resolução da equação autoconsistente para $\beta \varepsilon=2.5$. para $\beta \varepsilon=1.5$.

Figura 4.4: Gráficos com resolução da equação autoconsistente para diferentes valores de $\beta: 4,3.5,2.5$ e 1.5. À medida que diminuímos o valor de $\beta$, ou seja, aumentamos o valor da temperatura, o valor de $m^{*}$ tende a zero.

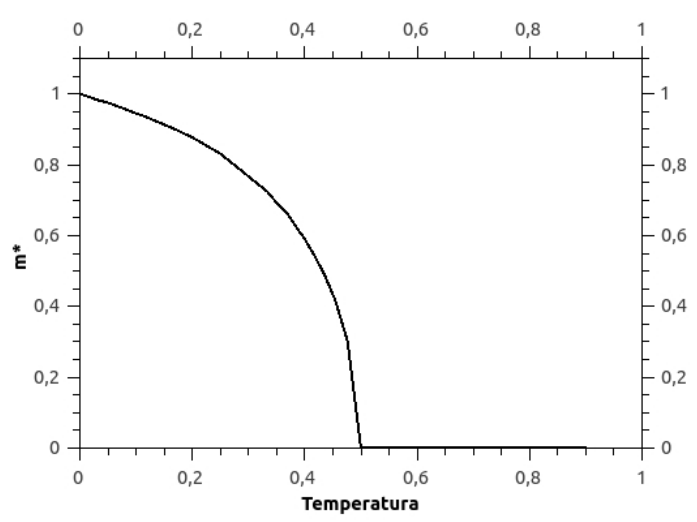

Figura 4.5: Gráfico de $m^{*}$ versus temperatura. 


$$
\begin{aligned}
e & =\frac{\partial(\beta f)}{\partial \beta}= \\
& =\frac{1}{2 \beta}+\frac{\varepsilon}{2}-\frac{y^{2}}{2 \beta \varepsilon}= \\
& =2 T+\frac{\varepsilon}{2}\left(1-m^{2}\right) .
\end{aligned}
$$

A partir das equações (4.53 e 4.54) podemos plotar a curva calórica do sistema, supondo $\varepsilon=1$, conforme é mostrado na figura (4.6).

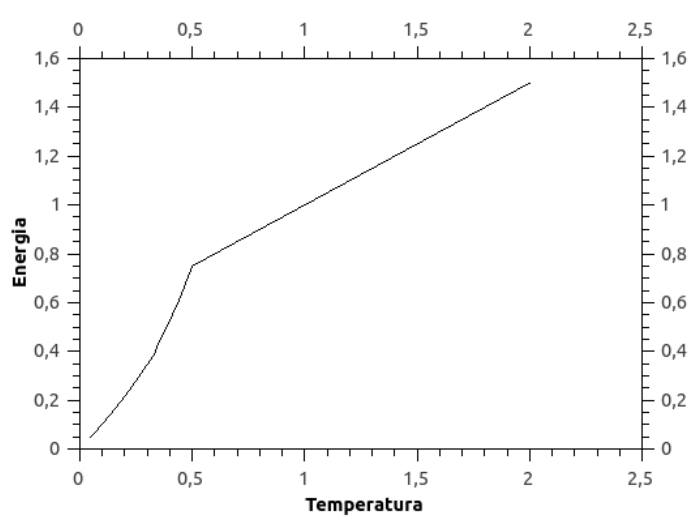

Figura 4.6: Curva calórica sistema HMF ensemble canônico.

Comparando esses resultados com os obtidos na sessão anterior, figuras (4.1) e (4.2), pode-se concluir que os ensembles canônico e microcanônico para o HMF 1-D são equivalentes.

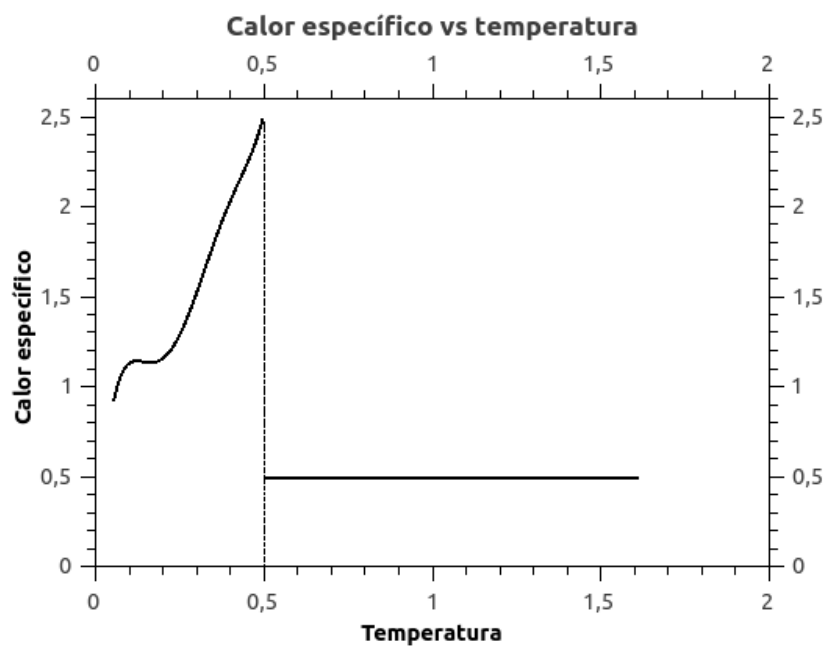

Figura 4.7: Calor específico versus temperatura para o HMF

Podemos plotar o gráfico do calor específico pela temperatura derivando a curva calórica, figura (4.6). Observa-se nesse caso uma descontinuidade finita no calor específico 
conforme mostra a figura (4.7).

Nesse capítulo calculamos a entropia e a energia de Helmholtz para o Hamiltoniano de Campo Médio em uma dimensão. Concluímos que os dois ensembles (microcanônico e canônico) são equivalentes, plotamos os gráficos da curva calórica dos dois ensembles e plotamos os gráficos que mostram a existência de transição contínua no sistema, com parâmetro de ordem $m$. No próximo capítulo vamos calcular o expoente crítico referente ao calor específico para esse sistema. 


\section{Capítulo 5}

\section{Resultados e conclusões}

Nesse capítulo vamos trabalhar com a energia de Helmholtz calculada para o Hamiltoniano de Campo Médio, equação (4.52). Usaremos lei de escala para comparar equações polinomiais com o objetivo de obter o expoente crítico $\alpha$.

\subsection{O cálculo do expoente crítico}

Considerando $f$ uma função homogênea podemos escrever, usando a teoria de escala:

$$
f\left(\lambda^{z} t, \lambda^{x} m\right)=\lambda f(t, m)
$$

Derivando os dois lados em relação a $\lambda$ :

$$
\frac{d f\left(\lambda^{z} t, \lambda^{x} m\right)}{d \lambda}=f(t, m)
$$

derivando pela regra da cadeia e sabendo que $\lambda$ pode ser qualquer número vamos consider $\lambda=1$ :

$$
t z \frac{\partial f}{\partial t}+m x \frac{\partial f}{\partial m}=f(t, m)
$$

A energia livre por partícula (equação 4.52) pode ser escrita, substituindo y por $m \beta \varepsilon$, que vem da equação (4.53),

$$
f(\beta, m)=-\frac{1}{2 \beta} \ln (2 \pi)+\frac{1}{2 \beta} \ln (\beta)+\frac{\varepsilon}{2}+\frac{1}{\beta}\left\{\frac{m^{2} \beta \varepsilon}{2}-\ln \left(2 \pi I_{0}(y)\right)\right\} .
$$

Definindo $k_{b}=1$, podemos reescrevemos a equação (5.3) na forma: 


$$
f(T, m)=-\frac{T}{2} \ln (2 \pi)-\frac{T}{2} \ln (T)+\frac{\varepsilon}{2}+T\left\{\frac{m^{2} \varepsilon}{2 T}-\ln \left(2 \pi I_{0}(y)\right)\right\} .
$$

Substituindo $T=T_{c}(1+t)$ na equação (5.4):

$$
\begin{aligned}
f(t, m)= & -\frac{T_{c}(1+t)}{2} \ln (2 \pi)-\frac{T_{c}(1+t)}{2} \ln \left(T_{c}(1+t)\right)+\frac{\varepsilon}{2}+\frac{m^{2} \varepsilon}{2}-T_{c}(1+t) \ln \left(2 \pi I_{0}(y)\right)= \\
= & -\frac{T_{c} t}{2} \ln (2 \pi)-\frac{T_{c}}{2} \ln (2 \pi)-\frac{T_{c} t}{2}\left[\ln \left(T_{c}\right)+\ln (1+t)\right]-\frac{T_{c}}{2}\left[\ln \left(T_{c}\right)+\ln (1+t)\right]+\frac{\varepsilon}{2}+ \\
& +\frac{m^{2} \varepsilon}{2}-T_{c} t \ln \left(2 \pi I_{0}(y)\right)-T_{c} \ln \left(2 \pi I_{0}(y)\right)= \\
= & {\left[-\frac{T_{c}}{2}\left(\ln \left(T_{c}\right)+3 \ln (2 \pi)\right)+\frac{\varepsilon}{2}\right]-\frac{T_{c}}{2}\left(\ln \left(T_{c}\right)+3 \ln (2 \pi)\right) t-\frac{T_{c}}{2} \ln (1+t)-} \\
& -\frac{T_{c}}{2} \ln (1+t) t+\frac{m^{2} \varepsilon}{2}-T_{c} \ln \left(I_{0}(y)\right)-T_{c} \ln \left(I_{0}(y)\right) t .
\end{aligned}
$$

Nomeando a relação:

$$
A=-\frac{T_{c}}{2}\left[\ln \left(T_{c}\right)+3 \ln (2 \pi)\right]+\frac{\varepsilon}{2},
$$

e as expansões (para $t \sim 0)$ :

$$
\begin{aligned}
& \ln (1+t)=t-\frac{t^{2}}{2}+\frac{1}{3} t^{3}-\frac{1}{4} t^{4}+\mathcal{O}(5) \\
& \frac{1}{(1+t)}=(1-t)+t^{2}-t^{3}+t^{4}+\mathcal{O}(5),
\end{aligned}
$$

$\mathrm{e}$

$$
\ln (I o(y))=\frac{1}{4} y^{2}-\frac{1}{64} y^{4}+\mathcal{O}(6) .
$$

O próximo passo será obter a expansão de $f(t, m)$ em primeira e segunda ordem.

\subsubsection{Energia de Hemlholtz com a primeira ordem das expansões}

Reescrevendo a equação (5.5)com a primeira ordem das expansões (5.7), (5.8) e (5.9):

$$
f(t, m)=A-\frac{T_{c}}{2}\left(\ln \left(T_{c}\right)+3 \ln (2 \pi)\right) t-\frac{T_{c}}{2} t-\frac{T_{c}}{2} t^{2}+\frac{m^{2} \varepsilon}{2}-T_{c} 0-T_{c} 0 t,
$$


reescrevendo:

$$
f(t, m)=A-\frac{T_{c}}{2}\left(\ln \left(T_{c}\right)+3 \ln (2 \pi)+1\right) t-\frac{T_{c}}{2} t^{2}+\frac{m^{2} \varepsilon}{2} .
$$

Aqui a primeira ordem da expansão (5.9) é igual a zero, pois sua expansão apresenta apenas termos de ordem superior a dois.

Usando a relação:

$$
B=\ln \left(T_{c}\right)+3 \ln (2 \pi)+1
$$

Reescrevemos (5.11) como:

$$
f(t, m)=A-\frac{T_{c}}{2} B t-\frac{T_{c}}{2} t^{2}+\frac{m^{2} \varepsilon}{2},
$$

derivando equação (5.12) por $m$ :

$$
\frac{\partial f}{\partial m}=\varepsilon m
$$

e por $t$ :

$$
\frac{\partial f}{\partial t}=\frac{-T_{c}}{2} B-T_{c} t
$$

Substituindo as derivadas na equação (5.2):

$$
\begin{gathered}
f(t, m)=t z\left\{\frac{-T_{c}}{2} B-T_{c} t\right\}+m x\{\varepsilon m\} \\
f(t, m)=-\frac{T_{c}}{2} B z t-T_{c} z t^{2}+\varepsilon x m^{2}
\end{gathered}
$$

Comparando a equação (5.12) com a equação (5.13) montamos a tabela 5.1:

Tabela 5.1: Termos para comparação da energia livre expandida em primeira ordem

\begin{tabular}{|l|l|l|}
\hline Ordem dos termos & Equação $(5.12)$ & Equação $(5.13)$ \\
\hline $\mathcal{O}(0)$ & $T_{c}[\ln (T c)+3 \ln (2 \pi)]=\varepsilon$ & não tem \\
\hline $\mathcal{O}(1) \mathrm{em} \mathrm{"} t$ " & $-\frac{T_{c}}{2} B$ & $-\frac{T_{c}}{2} B z$ \\
\hline $\mathcal{O}(2) \mathrm{em} " t "$ & $-\frac{T_{c}}{2}$ & $-T_{c} z$ \\
\hline $\mathcal{O}(2) \mathrm{em} " m$ " & $\frac{\varepsilon}{2}$ & $\varepsilon x$ \\
\hline
\end{tabular}

Dado que expandimos a energia de Helmholtz até a primeira ordem é essa ordem de análise que nos interessa, portanto, comparando apenas os termos de primeira ordem $(\mathcal{O}(1))$ na 
tabela (5.1), concluímos que:

- Comparando o termo, que acompanha $t$ :

$$
-\frac{T_{c}}{2} B=-\frac{T_{c}}{2} B z
$$

e portanto:

$$
z=1
$$

não é possível achar o valor para $x$ trabalhando apenas com a primeira ordem, portanto vamos tentar achar os valores de $x$ e $z$ expandindo a energia livre em segunda ordem.

\subsubsection{Energia de Helmholtz com a segunda ordem das expansões}

Agora, reescrevendo a equação (5.5)com a segunda ordem das expansões (5.7), (5.8) e (5.9):

$$
f(t, m)=A-\frac{T_{c}}{2}\left(\ln \left(T_{c}\right)+3 \ln (2 \pi)\right) t-\frac{T_{c}}{2}\left(t-\frac{t^{2}}{2}\right)-\frac{T_{c}}{2}\left(t-\frac{t^{2}}{2}\right) t+\frac{m^{2} \varepsilon}{2}-\frac{T_{c}}{4} y^{2}-\frac{T_{c}}{4} y^{2} t
$$

Substituindo $y$ por $m \beta \varepsilon$, que vem da equação (4.53):

$$
\begin{aligned}
f & =A-\frac{T_{c}}{2}\left(\ln \left(T_{c}\right)+3 \ln (2 \pi)\right) t-\frac{T_{c}}{2}\left(t-\frac{t^{2}}{2}\right)-\frac{T_{c}}{2}\left(t-\frac{t^{2}}{2}\right) t+\frac{m^{2} \varepsilon}{2}-\frac{T_{c}}{4} \frac{m^{2} \varepsilon^{2}}{T_{c}^{2}(1+t)^{2}}(1+t)= \\
& =A-\frac{T_{c}}{2} B t-\frac{T_{c}}{4} t^{2}+\frac{T_{c}}{4} t^{3}+\frac{m^{2} \varepsilon}{2}-\frac{m^{2} \varepsilon^{2}}{4 T_{c}}\left(1-t+t^{2}\right)= \\
& =A-\frac{T_{c}}{2} B t-\frac{T_{c}}{4} t^{2}+\frac{T_{c}}{4} t^{3}+\left(\frac{\varepsilon}{2}-\frac{\varepsilon^{2}}{4 T_{c}}\right) m^{2}+\left(\frac{\varepsilon^{2}}{4 T_{c}}\right) m^{2} t-\left(\frac{\varepsilon^{2}}{4 T_{c}}\right) m^{2} t^{2}
\end{aligned}
$$

Sendo $B=\ln \left(T_{c}\right)+3 \ln (2 \pi)+1$. Derivando equação (5.14) por $m$ :

$$
\frac{\partial f}{\partial m}=\left(\varepsilon-\frac{\varepsilon^{2}}{2 T_{c}}\right) m+\frac{\varepsilon^{2}}{2 T_{c}} m t-\frac{\varepsilon^{2}}{T_{c}} m t^{2}
$$

e por $t$ :

$$
\frac{\partial f}{\partial t}=-\frac{T_{c}}{2} B-\frac{T_{c}}{2} t+3 \frac{T_{c}}{4} t^{2}+\frac{\varepsilon^{2}}{4 T_{c}} m^{2}-\frac{\varepsilon^{2}}{2 T_{c}} m^{2} t
$$


Substituindo as equações (5.15) e (5.16) na equação (5.2):

$$
\begin{gathered}
f(t, m)=t z\left\{-\frac{T_{c}}{2} B-\frac{T_{c}}{2} t+3 \frac{T_{c}}{4} t^{2}+\frac{\varepsilon^{2}}{4 T_{c}} m^{2}-\frac{\varepsilon^{2}}{2 T_{c}} m^{2} t\right\}+ \\
+m x\left\{\left(\varepsilon-\frac{\varepsilon^{2}}{2 T_{c}}\right) m+\frac{\varepsilon^{2}}{2 T_{c}} m t-\frac{\varepsilon^{2}}{2 T_{c}} m t^{2}\right\} \\
f(t, m)=-\frac{T_{c}}{2} B z t-\frac{T_{c}}{2} z t^{2}+\frac{3 T_{c}}{4} z t^{3}+\left(\frac{\varepsilon^{2}}{4 T_{c}} z+\frac{\varepsilon^{2}}{2 T_{c}} x\right) m^{2} t-\left(\frac{\varepsilon^{2}}{2 T_{c}} z+\frac{\varepsilon^{2}}{2 T_{c}} x\right) m^{2} t^{2}- \\
-\left(\frac{\varepsilon^{2}}{2 T_{c}}-\varepsilon\right) x m^{2} .
\end{gathered}
$$

Olhando a equação (5.14) com a equação (5.17), montamos a tabela 5.2:

Tabela 5.2: Termos para comparação da energia livre expandida em segunda ordem

\begin{tabular}{|l|l|l|}
\hline Ordem dos termos & Equação $(5.14)$ & Equação $(5.17)$ \\
\hline $\mathcal{O}(0)$ & $T_{c}[\ln (T c)+3 \ln (2 \pi)]=\varepsilon$ & não tem \\
\hline $\mathcal{O}(1), \mathrm{em}$ " $t$ " & $-\frac{T_{c}}{2} B$ & $-\frac{T_{c}}{2} B z$ \\
\hline $\mathcal{O}(2) \mathrm{em} " t$ " & $-\frac{T_{c}}{4}$ & $-\frac{T_{c}}{2} z$ \\
\hline $\mathcal{O}(2) \mathrm{em}$ " $m$ " & $\left(\frac{\varepsilon}{2}-\frac{\varepsilon^{2}}{4 T_{c}}\right)$ & $\left(-\frac{\varepsilon^{2}}{2 T_{c}}+\varepsilon\right) x$ \\
\hline $\mathcal{O}(3) \mathrm{em} \mathrm{"} m t^{\prime}$ & $\frac{\varepsilon^{2}}{4 T_{c}}$ & $\left(\frac{\varepsilon^{2}}{4 T_{c}} z+\frac{\varepsilon^{2}}{2 T_{c}} x\right)$ \\
\hline $\mathcal{O}(4) \mathrm{em} \mathrm{"} m t^{\prime}$ & $-\frac{\varepsilon^{2}}{4 T_{c}}$ & $-\left(\frac{\varepsilon^{2}}{2 T_{c}} z+\frac{\varepsilon^{2}}{2 T_{c}} x\right)$ \\
\hline
\end{tabular}

Comparando apenas os termos de segunda ordem em $t$ e em $m$, pois dado que expandimos a energia de Helmholtz até essa ordem é essa ordem de análise que nos interessa, concluímos que:

- A comparação do termo que acompanha $t^{2}$ :

$$
-\frac{T_{c}}{4} t^{2}=-\frac{T_{c}}{2} z t^{2}
$$

que nos leva à relação

$$
z=\frac{1}{2}
$$

- A comparação do termpo que acompanha $m^{2}$ : 


$$
\left(-\frac{\varepsilon^{2}}{2 T_{c}}+\varepsilon\right) x=\left(\frac{\varepsilon}{2}-\frac{\varepsilon^{2}}{4 T_{c}}\right)
$$

que nos leva à relação

$$
x=\frac{1}{2}
$$

Podemos obervar na tabela (5.3) os valores de $x$ e $z$ dependendo da ordem das expansões.

Tabela 5.3: Valores de x e z para primeira e segunda ordem das expansões de $f(t, m)$

\begin{tabular}{|l|l|l|}
\hline & Primeira ordem & Segunda ordem \\
\hline $\mathrm{z}$ & 1 & $\frac{1}{2}$ \\
\hline $\mathrm{x}$ & Não é possível determinar & $\frac{1}{2}$ \\
\hline
\end{tabular}

Portanto, pela equação $(2.25)$, mas agora para $m$ constante, $c_{m} \sim t^{\left(\frac{1}{z}-2\right)}$, temos:

$$
\alpha=2-\frac{1}{z}
$$

e pelo valor achado pela segunda ordem das expansões na equação (5.18), o valor de $\alpha$ é:

$$
\alpha=0 .
$$

Esse valor concorda com o previsto pela teoria de Landau, concorda com o gráfico achado para o comportamento do calor específico, figura (4.7) e concorda também com o valor achado pela derivação da energia de Helmholtz no Apêndice A.

\subsection{Conclusões}

Considerando que o sistema estudado possui interação de longo alcance e foi afirmado que esses sistemas nem sempre possuem equivalência entre os ensembles, a primeira conclusão que obtivemos foi que os dois ensembles desse sistema são equivalentes. E não possuem calor específico negativo, conclusões que podem ser tiradas da análise das curvas calóricas dos dois ensembles.

Calculamos a energia livre de Helmholtz de um sistema com interação de longo alcance (HMF) e plotamos os gráficos do calor específico (4.3) e (4.7) que mostram uma 
descontinuidade no ponto crítico, o que está de acordo com o previsto pela Teoria de Landau. E ao analisar a derivada da energia livre de Helmholtz no Apêndice A observamos que o calor específico também é descontínuo no ponto crítico, reproduzindo o esperado pela Teoria de Landau.

Usando teoria de escala, observamos que, em primeira ordem de aproximação não conseguimos achar valor para $x$, apenas para $z$, o que mostra ser necessário uma ordem de expansão maior. Em segunda ordem de aproximação temos que o valor do expoente crítico para o calor específico é igual ao previsto pela Teoria de Landau, sendo a primeira vez que ele é calculado analiticamente para o sistema HMF pela teoria de escala. Por essa forma de análise o calor específico se mostra descontínuo no ponto crítico. 


\section{Apêndice A}

\section{Derivação da energia livre}

Considerando a energia de Helmholtz do sistema HMF:

$$
\begin{aligned}
f(T, m) & =-\frac{T}{2} \ln (2 \pi)-\frac{T}{2} \ln (T)+\frac{\varepsilon}{2}+T\left\{\frac{m^{2} \varepsilon}{2 T}-\ln \left(2 \pi I_{0}(y)\right)\right\}= \\
& =-\frac{T}{2} \ln (2 \pi)-\frac{T}{2} \ln (T)+\frac{\varepsilon}{2}+\frac{m^{2} \varepsilon}{2}-T \ln (2 \pi)-T \ln \left(I_{0}(y)\right)= \\
& =-\frac{T}{2} \ln (2 \pi)-\frac{T}{2} \ln (T)+\frac{\varepsilon}{2}+\frac{y^{2} T^{2}}{2 \varepsilon}-T \ln (2 \pi)-T \ln \left(I_{0}(y)\right)
\end{aligned}
$$

substituindo $m$ por $\frac{y T}{\varepsilon}$.

Sabemos que o calor específico do sistema pode ser calculado pela equação (1.1), com $m$ constante, na região crítica $T=T_{c}$ então para estudar a divergência ou descontinuidade do calor específico na transição de fase contínua vamos observar apenas o comportamento da segunda derivada de $f$ em $T$ :

$$
c_{m}=-T_{c}\left(\frac{\partial^{2} f}{\partial T^{2}}\right)_{m}
$$

E lembrando que o calor específico se comporta de acordo com a equação (2.1), para $m$ constante:

$$
c_{m} \sim t^{-\alpha}
$$

vamos derivar a equação (A.1) para achar a dependência de $c_{m} \operatorname{com} t$ na região crítica, ou seja, $m \sim 0$ e $t \sim 0$ : 


$$
\begin{aligned}
c_{m} & =-T_{c}\left(\frac{\partial^{2} f}{\partial T^{2}}\right)_{m}= \\
& =-T_{c}\left[\frac{1}{2 T}-\frac{y^{2}}{\varepsilon}\right]= \\
& =-T_{c}\left[\frac{1}{2 T}-\frac{m^{2} \varepsilon}{T^{2}}\right]= \\
& =-\frac{1}{2(1+t)} \sim t^{0}
\end{aligned}
$$

lembrando que $T=T_{c}(1+t)$.

Portanto, percebemos que há uma descontinuidade no ponto crítico e o valor de $\alpha=0$, o que é previsto pela Teoria de Landau. 


\section{Referências Bibliográficas}

[1] Hidetoshi Nishimori and Gerardo Ortiz. Elements of Phase Transitions and Critical Phenomena. Oxford, Oxford University press, 2010.

[2] Moshe Gitterrnan and Vivian (Hairn) Halpern. Phase transitions - A Brief Account with Modern Applications. Massachusetts, World Scientific Publishing Co. Pte. Ltd., 2004 .

[3] Herbert B. Callen. Thermodynamics and an Introduction to Thermostatistics. Singapura, John wiley \& sons, 1985.

[4] H. Eugene Stanley. Scaling, universality, and renormalization: Three pillars of modern critical phenomena. Reviews Of Modern Physics, 71(2):358-366, 1999.

[5] Ennio Arimondo Thierry Dauxois, Stefano Ruffo and Martin Wilkens. Dynamics and thermodynamics os systems with long range interactions: An introduction. ArXiv, 2002.

[6] A. J. Fischer e M. E. J. Newman J. J. Binney, N. J. Dowrick. The theory of Critical Phenomena. Oxford, Oxford University Press, 1992.

[7] Navinder Singh. Thermodynamical phase transitions, the mean-field theories, and the renormalization (semi)group: A pedagogical introduction. ArXiv, 2014.

[8] Moises Fabiano Pereira da Silva Junior. Comparação de métodos computacionais para o estudo da termodinâmica de sistemas com interações de longo alcance. Dissertação de Mestrado apresentada ao Instituto de Física da Universidade de Brasília, 2016.

[9] H. Eugene Stanley. Introduction to phase transitions and critical phenomena. Oxford, Oxford University Press, 1987.

[10] Julia M. Yeomans. Statistical mechanics of phase transitions. Oxford, Oxford University Press, 1992. 
[11] A. E. Guggenheim. The principle of corresponding states. Chemical Physics, $13(7): 253,1945$.

[12] R. K. Pathria and Paul D. Beale. Statistical Mechanics. Massachusetts, Elsevier Ltd, 2011.

[13] João Marcos Batista de Souza Maciel. Método De Monte Carlo Microcanônico Aplicado À Sistemas Auto-Gravitantes. Projeto de doutorado apresentado ao Instituto de Física da Universidade de Brasília, 2013.

[14] Leo P. Kadanoff. Relating theories via renormalization. Studies in History and Philosophy of Science Part B: Studies in History and Philosophy of Modern Physics, 44(1):22-39, 2013.

[15] Mickael Antoni and Stefano Ruffo. Clustering and relaxation in hamiltonian longrange dynamics. Physical Review E, 52(3):2361, 1995.

[16] Alessandro Campa, Thierry Dauxois, and Stefano Ruffo. Statistical mechanics and dynamics of solvable models with long-range interactions. Physics Reports, 480(3):57159, 2009. 